\title{
ON $q$-GALOIS EXTENSIONS OF SIMPLE RINGS
}

\author{
HISAO TOMINAGA
}

To the memory of Professor TAdasi Nakayama

In 1952, the late Professor T. Nakayama succeeded in constructing the Galois theory for finite dimensional simple ring extensions [7]. And, we believe, the theory was essentially due to the following proposition: If a simple ring $A$ is Galois and finite over a simple subring $B$ then $A$ is $B^{\prime}-A$-completely reducible for any simple intermediate ring $B^{\prime}$ of $A / B$ [7, Lemmas 1.1 and $1.2^{1)}$ ]. Moreover, as was established in [5], Nakayama's idea was still efficient in considering the infinite dimensional Galois theory of simple rings.

In this paper, we shall present first such a generalization of the proposition stated above that contains [5, Lemma 2] as well. And then, by the aid of this generalization, several facts obtained in [6] and [8] for division rings will be extended to simple rings. In fact, under the assumption that a simple ring extension in question is $h-q$-Galois and left locally finite, many important results previously obtained in [2]-[10] can be unified.

Throughout the present paper, $A=\sum_{1}^{n} D e_{i j}$ will represent a simple ring where $E=\left\{e_{i j}\right.$ 's $\}$ is a system of matrix units and $D=V_{A}(E)$ a division ring, and $B$ a simple subring of $A$ containing the identity 1 of $A$. And we use the following conventions: $V$ and $H$ mean $V_{A}(B)$ and $V_{A}^{2}(B)=V_{A}\left(V_{A}(B)\right)$, respectively. If $H$ is a simple ring, we set $H=\sum K d_{i k}$ where $\Delta=\left\{d_{\iota^{\prime}}\right.$ 's $\}$ is a system of matrix units and $K=V_{H}(\Delta)$ a division ring. If $T$ is a regular subring of $A$ containing $B, \mathbb{B}(T, A / B)$ will mean the set of all the $B$-(ring) isomorphisms of $T$ onto regular subrings of $A$. And finally, $A / B$ is said to be $h$ Galois $^{2 \prime}$ if $B$ is regular and $B A_{r}$ is dense in $\operatorname{Hom}_{B_{l}}(A, A)$, where $B$ is the

\footnotetext{
Received April 26, 1965.

1) These lemmas were stated under the weaker assumption that $A / B$ is (finite and) weakly normal.

2) In [4], $A / B$ was defined to be $h$-Galois if (i) $B$ is regular, (ii) $A$ is Galois over $B^{\prime}$ and $V_{A}^{2}\left(B^{\prime}\right)$ is simple for any regular subring $B^{\prime}$ of $A$ left finite over $B$, and (iii) $A^{\prime}=V_{A}^{2}\left(A^{\prime}\right)$ and $\left[A^{\prime}: H\right]_{l}=\left[V: V_{A}\left(A^{\prime}\right)\right]_{r}$ for every regular subring $A^{\prime}$ of $A$ left finite over $H$, and it (Continued on next page)
} 
group of all the $B$-automorphisms of $A$. As to other notations and terminologies used here, we follow [4] and [5].

The following propositions previously known will play important roles in the present study.

Proposition 1. Let $B^{\prime}$ be a subring of $A$ containing 1 of $A, V^{\prime}=V_{A}\left(B^{\prime}\right)$ and $H^{\prime}=V_{A}^{2}\left(B^{\prime}\right)$.

(a) If $A$ is $B^{\prime} \cdot V^{\prime}-A$-irreducible, then $A$ is homogeneously completely reducible as $B^{\prime}-A$-module and as $V^{\prime}-A$-module, both $V^{\prime}$ and $H^{\prime}$ are simple rings, $\left[A \mid B_{l}^{\prime} \cdot A_{r}\right]$ $=\left[V^{\prime} \mid V^{\prime}\right]$ and $\left[A \mid V_{l}^{\prime} \cdot A_{r}\right]=\left[H^{\prime} \mid H^{\prime}\right]^{3)}$.

(b) If $B^{\prime}$ is an intermediate ring of $A / B$ left (resp. right) finite over $B$ and $A$ is $B^{\prime} \cdot V^{\prime}-A$-irreducible (resp. $A-B^{\prime} \cdot V^{\prime}$-irreducible), then $\left[V: V^{\prime}\right]_{r} \leq\left[B^{\prime}: H^{*}\right]_{\text {l }}$ (resp. $\left[V: V^{\prime}\right]_{l} \leq\left[B^{\prime}: H^{*}\right]_{r}$ ) for any simple intermediate ring $H^{*}$ of $H \cap B^{\prime} / B$.

(c) If $B^{\prime}$ is an intermediate ring of $A / B[E]$ left (resp. right) finite over $B$ and $A$ is left (resp. right) locally finite over $B$, then $\left[V: V^{\prime}\right]_{l} \leq\left[B^{\prime}: B\right]_{l}$ (resp. $\left.\left[V: V^{\prime}\right]_{r} \leq\left[B^{\prime}: B\right]_{r}\right)$. ([2, Lemma 1 and Cor. 2].)

Proposition 2. Let $A$ be outer Galois and left locally finite over $B$, and $A^{\prime}$ an intermediate ring of $A / B$.

(a) $A^{\prime}$ is simple, $A / A^{\prime}$ is (two-sided) locally finite, and each $B$-(ring) isomorphism of $A^{\prime}$ into $A$ can be extended to an element of $\mathbb{B}$.

(b) $A / B$ is $h$-Galois, and there exists a 1-1 dual correspondence between closed subgroups of $B$ and intermediate rings of $A / B$, in the usual sense of Galois theory.

(c) If $\left[A^{\prime}: B\right]_{l}<\propto$ then $\left[A^{\prime}: B\right]_{l}=\left[A^{\prime}: B\right]_{r}=\#\left(\mathfrak{g} \mid A^{\prime}\right)$ for any Galois group $\mathfrak{S}$ of $A / B$. ([3, Th. 1.1], [3, Cor. 1.4], [4, Lemma 1.8] and [9].)

Proposition 3. Let $A$ be Galois over $B$ with a regular Galois group 5 , and $H$ a simple ring left locally finite over $B . \quad$ And let $T$ be an intermediate ring of $A / B$ such that $[T: B]_{l}<\infty$ and $A$ is $T$-A-irreducible.

(a) If $[T: H \cap T]_{l}=\left[V: V_{A}(T)\right]_{r}$ then $\operatorname{Hom}_{B_{l}}(T, A)=(\mathfrak{g} \mid T) A_{r}$ and $\$(T$ $=\mathfrak{S} \mid T$.

was shown that if $A / B$ is $h$-Galois and left locally finite then $\left(s A_{r}\right.$ is dense in $\operatorname{Hom}_{B}(A$, $A$ ). And more recently, in [2], T. Nagahara has shown the converse implication. However, one will see later the converse implication to be true even under a somewhat weakened assumption. (Cf. Ths. 2 and 8.)

3) $\left[A \mid B_{l}^{\prime} \bullet A r\right]$ and $\left[V^{\prime} \mid V^{\prime}\right]$ denote the length of the composition series of the $B^{\prime}-A$ module $A$ and the length of the composition series of the $V^{\prime}$-module $V^{\prime}$ (the capacity of the simple ring $V^{\prime}$ ), respectively. 
(b) If $T^{\prime}=J(\&(T), A)$ then $\left[H \cap T^{\prime}: B\right]<\infty$. ([4, Lemma 3.1] and $[5$, Lemma 5].)

1. Preliminaries. The present section starts with the following brief lemma.

Lemma 1. Let $B^{\prime}$ be a simple intermediate ring of $A / B$ with $\left[B^{\prime} \mid B^{\prime}\right]=n(=$ capacity of $A$ ). If $a$ is an arbitrary element of $A$ and $T$ an arbitrary simple intermediate ring of $A / B^{\prime}$ then $\left[a B^{\prime} \mid B^{\prime}\right] \geq[a T \mid T] . \quad A n d$, if $A / B$ is left locally finite and $\left[B^{\prime}: B\right]_{l}<\infty$ then there exists an intermediate ring $B^{\prime \prime}$ of $A / B^{\prime}$ such that $\left[B^{\prime \prime}: B\right]_{l}<\infty$ and $\left[a B^{\prime \prime} \mid B^{\prime \prime}\right]=[a A \mid A]$.

Proof. Without loss of generality, we may assume that $B^{\prime}$ contains $E$ and $a B^{\prime}=\sum_{1}^{n} a e_{i i} B^{\prime}=\oplus_{1}^{m} a e_{i i} B^{\prime}\left(m=\left[a B^{\prime} \mid B^{\prime}\right]\right) . \quad$ As each $e_{i i} T=e_{i i} B^{\prime} T$ is a minimal right ideal of $T, a T=a B^{\prime} T=\sum_{1}^{m} a e_{i i} T$ implies then $[a T \mid T] \leq m$. Now, the rest of the proof will be obvious.

The proof of the next lemma proceeds in the usual way (cf. [4]), and may be omitted.

Lemma 2. Let $B^{\prime}$ be a simple intermediate ring of $A / B$ with $\left[B^{\prime} \mid B^{\prime}\right]=n, \alpha$ and $\beta$ elements of $\mathbb{S}\left(B^{\prime}, A / B\right)$, and $\mathfrak{S}$ a subset of $\left(\mathbb{S}\left(B^{\prime}, A / B\right)\right.$.

(a) $\alpha A_{r}$ is $B_{r}^{\prime}-A_{r}$-irreducible and $\alpha$ is linearly independent over $A_{r}$.

(b) Let $\mathrm{m}$ be a $B_{r}^{\prime}-A_{r}$-submodule of $\mathfrak{S} A_{r} . \quad \mathrm{m}$ is $B_{r}^{\prime}-A_{r}$-irreducible if and only if $\mathrm{in}=\sigma u_{l} A_{r}$ with some $\sigma \in \mathfrak{S}$ and some non-zero $u \in V$.

(c) $\alpha A_{r}$ is $B_{r}^{\prime}-A_{r}$-isomorphic to $\beta A_{r}$ if and only if $\alpha=\beta \tilde{u}$ with some $u \in V$. (the multiplicative group of the regular elements of $V$ ), and so if $\alpha$ is contained in $\mathfrak{S}_{\text {g }}$ then $\alpha=\sigma \tilde{v}$ with some $\sigma \in \mathfrak{S}$ and $v \in V^{\cdot}$.

We consider here the following conditions :

(1) $\operatorname{Hom}_{B l}\left(B^{\prime}, A\right)=\$\left(B^{\prime}, A / B\right) A_{r}$ for any regular intermediate ring $B^{\prime}$ of $A / B$ with $\left[B^{\prime}: B\right]_{l}<\infty$.

(1') $\operatorname{Hom}_{B_{r}}\left(B^{\prime}, A\right)=\mathbb{B}\left(B^{\prime}, A / B\right) A_{l}$ for any regular intermediate ring $B^{\prime}$ of $A / B$ with $\left[B^{\prime}: B\right]_{r}<\infty$.

(2) $\mathbb{B}\left(B_{1}, A / B\right) \mid B_{2} \subseteq \mathbb{S}\left(B_{2}, A / B\right)$ for any regular subrings $B_{1} \supseteq B_{2}$ of $A$ containing $B$ with $\left[B_{1}: B\right]_{l}<\infty$.

(2') $\&\left(B_{1}, A / B\right) \mid B_{2} \subseteq \mathbb{B}\left(B_{2}, A / B\right)$ for any regular subrings $B_{1} \supseteq B_{2}$ of $A$ containing $B$ with $\left[B_{1}: B\right]_{r}<\infty$.

Remark 1. If the condition (1) is satisfied, then $J\left(\$\left(B^{\prime}, A / B\right), B^{\prime}\right)=B$ 
for any regular intermediate ring $B^{\prime}$ of $A / B$ with $\left[B^{\prime}: B\right]_{l}<\infty$. In fact, if $b^{\prime}$ is an arbitrary element of $J\left(\&\left(B^{\prime}, A / B\right), B^{\prime}\right)$ not contained in $B$ then $T=$ $B\left[b^{\prime}\right]$ is a subring of $B^{\prime}$ properly containing $B$. Since $\operatorname{Hom}_{\nu_{l}}\left(B^{\prime}, A\right)=\mathbb{B}\left(B^{\prime}\right.$, $A / B) A_{r}$, we have $\operatorname{Hom}_{B_{l}}(T, A)=\operatorname{Hom}_{B_{l}}\left(B^{\prime}, A\right) \mid T=\left(\mathbb{B}\left(B^{\prime}, A / B\right) \mid T\right) A_{r}=$ 1|T) $A_{r}$, whence it follows a contradiction $[T: B]_{l}=1$.

Now, we shall prove our first theorem which contains evidently the proposition cited at the opening as well as [5, Lemma 2].

Theorem 1. Let $A / B$ be left locally finite, and the condition (1) satisfied. If $T$ is a simple intermediate ring of $A / B$ with $[T: B]_{l}<\infty$ then $A$ is $T$-Acompletely reducible. In particular, if $T$ is a regular subring of $A$ with $[T: B]_{l}$ $<\infty$ then $A$ is homngeneously $T$-A-completely reducible with $\left[A \mid T_{l} \cdot A_{r}\right]=$ $\left[V_{A}(T) \mid V_{A}(T)\right]$ and $T$ is f-regular.

Proof. Let $M$ be an arbitrary minimal $T$-A-submodule of $A$ such that thh composition series of $M$ as right $A$-module is of the shortest length among minimal $T$ - $A$-submodules of $A$. Then, $M=e A$ with a non-zero idempotent $e$. In virtue of Lemma 1 , we can find an intermediate ring $T^{*}$ of $A / T[E, e]$ with $\left[T^{*}: B\right]_{l}<\infty$ and $\left[e T^{*} \mid T^{*}\right]=[e A \mid A]$. One may remark here that $T e T^{*}=$ $e T^{*}$. In fact, for each $t \in T$ there exists some $a \in A$ with $e a=t e \in T^{*}$, so that $t e=e \cdot e a \in e T^{*}$. By Lemma $2(\mathrm{a}), \operatorname{Hom}_{B_{l}}\left(T^{*}, A\right)=\$\left(T^{*}, A / B\right) A_{r}$ is $T_{r}^{*}-A_{r^{-}}$ completely reducible. Accordingly, the $T_{r}^{*}-A_{r}$-module $\operatorname{Hom}_{l^{\prime} l}\left(T^{*}, A\right)=\oplus_{1}^{t} \mathfrak{M}_{j}$ with $T_{r}^{*}-A_{r}$-irreducible $\mathfrak{M}_{j}$. By Lemma $2(\mathrm{~b}), \mathfrak{M}_{j}=\sigma_{j} u_{\jmath l} A_{r}$ with some $\sigma_{j} \in$ $\mathbb{B}\left(T^{*}, A / B\right)$ and non-zero $u_{j} \in V$. Since $\mathfrak{M}_{j} \subseteq \operatorname{Hom}_{T_{l}}\left(T^{*}, A\right)$ and $T e T^{*}=e T^{*}(\subseteq$ $\left.T^{*}\right)$, each $M_{j}=(T e) \mathbb{M}_{j}$ is a $T$ - $A$-submodule of $A$. Further, there holds $M_{j}=$ $u_{j} \cdot(T e)_{\sigma_{j}} \cdot A=u_{j} \cdot\left(T e T^{*}\right)_{\sigma_{j}} \cdot A=u_{j} \cdot\left(e T^{*}\right)_{\sigma_{j}} \cdot A=u_{j} \cdot e_{\sigma_{j}} \cdot A$, whence it follows $\left[M_{j} \mid A\right]$ $=\left[u_{j} \bullet e_{j} \cdot A \mid A\right] \leq\left[e \sigma_{j} \cdot A \mid A\right] \leq\left[e_{\sigma_{j}} \cdot T^{*} \sigma_{j} \mid T^{*} \sigma_{j}\right]=\left[e T^{*} \mid T^{*}\right]=[M \mid A]$ by Lemma 1 . Recalling here that $[M \mid A]$ is the least, we see that each $M_{j}$ is either 0 or $T$ - $A$-irreducible. Finally, noting that $A$ is $T_{l} \cdot \operatorname{Hom}_{\Gamma_{l}}(A, A)$-irreducible, there holds $A=e\left(T_{l} \cdot \operatorname{Hom}_{T_{l}}(A, A)\right)=(T e) \operatorname{Hom}_{T_{l}}\left(T^{*}, A\right)=(T e) \sum \vartheta l_{j}=\sum M_{j}$, which proves evidently the complete reducibility of $A$ as $T$ - $A$-module. Now, the latter assertion will be evident by Prop. 1 (b).

The next has been proved in [2] and [5]. Nevertheless, according to the idea in [7], we shall present here another proof that needs only Lemma 2 and Th. 1. 
Corollary 1. Let $A$ be left locally finite over a regular subring $B$, and $\$_{2} A_{r}$ is dense in $\operatorname{Hom}_{B_{l}}(A, A)$ for an automorphism group $\mathscr{S}$ containing $\tilde{V}$. If $B^{\prime}$ is a regular intermediate ring of $A / B$ with $\left[B^{\prime}: B\right]_{l}<\infty$ then $\mathscr{H}_{(}\left(B^{\prime}\right) A_{r}$ is dense in $\operatorname{Hom}_{B^{\prime}}(A, A)$ and $J\left(S_{2}\left(B^{\prime}\right), A\right)=B^{\prime}$.

Proof. Let $T$ be an arbitrary intermediate ring of $A / B^{\prime}[E]$ with $[T: B]_{l}$ $<\infty$. Evidently, $\operatorname{Hom}_{B^{\prime} l}(T, A)$ is a $T_{r}-A_{r}$-submodule of $\operatorname{Hom}_{P_{l}}(T, A)=$ $(\mathfrak{S} \mid T) A_{r}$. And then, by Lemma $2(\mathrm{~b}), \operatorname{Hom}_{B^{\prime} l}(T, A)=\oplus\left(\sigma_{i} u_{i l} \mid T\right) A_{r}$ with some $\sigma_{i} \in \mathfrak{S}$ and non-zero $u_{i} \in V$. In general, if $\tau w_{l} \mid T(\tau \in \mathfrak{S}, w \in V)$ is contained in $\operatorname{Hom}_{B^{\prime} \iota}(T, A)$, one will easily see that $\tau w_{l}$ is contained in $V_{\mathfrak{g}}\left(B_{l}^{\prime}\right)(\mathfrak{Q}=\operatorname{Hom}(A, A))$. Now, let $\sigma u_{l}$ be an arbitrary $\sigma_{i} u_{i l}$. Since $A$ is homogeneously $B^{\prime}$ - $A$-completely reducible by Th. 1 , a standard argument enables us to find such an inversible element $\nu \in V_{\mathfrak{A}}\left(B_{l}^{\prime}\right)$ that $a_{r} \nu=\nu(a \sigma)_{r}$ for all $a \in A$. As $\nu^{-1} \sigma u_{l}$ is then contained in $V_{\mathfrak{A}}\left(B_{l}^{\prime} \cdot A_{r}\right)=V_{A}\left(B^{\prime}\right)_{l}, \sigma u_{l}=\nu v_{1 l}+\cdots+\nu v_{m l}$ with some $v_{j} \in V_{A}\left(B^{\prime}\right)^{\prime}$. Noting that $T$ contains $E$, one will easily see that every $\left(\nu v_{j l} \mid T\right) A_{r}$ is a $T_{r}-A_{r^{-}}$ irreducible submodule of $\operatorname{Hom}_{B^{\prime} l}(T, A)$, so that $\left(\nu v_{j l} \mid T\right) A_{r}=\left(\tau w_{j l} \mid T\right) A_{r}$ with some $\tau \in \mathfrak{B}$ and $w_{j} \in V$ (Lemma 2). We have then $A=v_{j} A=v_{j} \cdot A \nu=v_{j} \cdot(T \cdot$ $\left.A \sigma^{-1}\right)_{\nu}=v_{j} \cdot T \nu \cdot A=T\left(\nu v_{j l} \mid T\right) A_{r}=T\left(\tau w_{j l} \mid T\right) A_{r}=w_{j} \cdot T \tau \cdot A=w_{j} A$, whence it follows $w_{j} \in V^{*}$. Hence, $\tau \widetilde{w}_{j}=\tau w_{j l} w_{j r}^{-1}$ is contained in $V_{\mathfrak{Q}}\left(B_{l}^{\prime}\right) \cap \mathfrak{S}_{\mathfrak{g}}=\mathfrak{S}_{(}\left(B^{\prime}\right)$. It follows therefore $\operatorname{Hom}_{B^{\prime} l}(T, A)=\left(\mathscr{S}^{\prime}\left(B^{\prime}\right) \mid T\right) A_{r}$, which forces $\mathfrak{S}_{(}\left(B^{\prime}\right) A_{r}$ to be dense in $\operatorname{Hom}_{B^{\prime} \iota}(A, A)$. Finally, to be easily verified, $B_{l}=V_{\mathfrak{U}}^{2}\left(B_{l}\right)=V_{\mathfrak{A l}}\left(\mathfrak{S}_{\mathfrak{g}} A_{r}\right)$, which implies $J(\mathfrak{g}, A)=B$. And hence, by the fact proved above, $J\left(\mathfrak{g}\left(B^{\prime}\right), A\right)$ $=B^{\prime}$.

Patterning after the proof of $[2$, Lemma 2$]$, we readily obtain the next:

Lemma 3. Let $H$ be simple, and $T$ an intermediate ring of $A / B[\Delta]$. If there exists an automorphism group $\mathfrak{S}$ of $H[T]$ with $J(\mathfrak{g}, H[T])=T$ and $H \mathfrak{B}=H$, and if $H \cap T$ is simple, then $T$ is linearly disjoint from $H$.

The following proposition is a part of [2, Th. 1]. However, for the sake of completeness, we shall give here the proof.

Propositron 4. If $B$ is a regular subring of $A$, the following conditions are equivalent to each other:

(A) $A$ is $h$-Galois and left locally finite over $B$.

$\left(\mathrm{A}^{\prime}\right) \mathscr{S}_{l}$ l is dense in $\operatorname{Hom}_{B_{r}}(A, A)$ and $A / B$ is right locally finite.

(B) $A$ is Galois and left locally finite over $B$, and $B \cdot V$-A-irreducible. 
(B') $A$ is Galois and right locally finite over $B$, and $A-B \cdot V$-irreducible.

(C) $A$ is Galois and left locally finite over $B$, and $A-B \cdot V$-irreducible.

$\left(\mathrm{C}^{\prime}\right) A$ is Galois and right locally finite over $B$, and $B \cdot V$ A-irreducible.

Proof. $(\mathrm{A}) \Longrightarrow(\mathrm{B})$ is obvious by Th. 1 and Cor. 1 . Next, we shall prove (B) $\Longrightarrow\left(\mathrm{C}^{\prime}\right) \Longrightarrow\left(\mathrm{A}^{\prime}\right)$. As $A$ is $B \cdot V \cdot A$-irreducible, $H$ is simple by Prop. 1 (a). For an arbitrary intermediate ring $T$ of $A / B[E, \Delta]$ with $[T: B]_{r}<\infty$, we set $T^{\prime}=J(B(T), A)$ and $H^{\prime}=H \cap T^{\prime}$. Then, $\left[V: V_{A}\left(T^{\prime}\right)\right]_{l}=\left[V: V_{A}(T)\right]_{l} \leq$ $[T: B]_{r}$ by Prop. $1(\mathrm{~b})$, and so Lemma 3 and Prop. 1 (b) imply $\left[T^{\prime}: H^{\prime}\right]_{r}$ $=\left[T^{\prime} \cdot H: H\right]_{r} \leq\left[V_{A}^{2}\left(T^{\prime}\right): H\right]_{r} \leq\left[V: V_{A}\left(T^{\prime}\right)\right]_{l}<\infty$. On the other hand, noting that $A$ is $A-T^{\prime}$-irreducible, Prop. 1 (b) yields also $\left[V: V_{A}\left(T^{\prime}\right)\right]_{l} \leq\left[T^{\prime}: H^{\prime}\right]_{r}$ $<\infty$. Combining those above, we obtain $\left[T^{\prime}: H^{\prime}\right]_{r}=\left[V: V_{A}\left(T^{\prime}\right)\right]_{l}$. Since $\left[T^{\prime}: B\right]_{r}=\left[T^{\prime}: H^{\prime}\right]_{r} \cdot\left[H^{\prime}: B\right]<\infty$ by Prop. $3(\mathrm{~b})$, the proposition symmetric to Prop. 3 (a) yields $\operatorname{Hom}_{F_{r}}\left(T^{\prime}, A\right)=\left(\$ \mid T^{\prime}\right) A_{l}$, which proves $\left(\mathrm{C}^{\prime}\right) \Rightarrow\left(\mathrm{A}^{\prime}\right)$. In case the condition (B) is satisfied, for an arbitrary intermediate ring $T$ of $A / B[E, \Delta]$ with $[T: B]_{l}<\infty$ there holds $\left[V: V_{A}(T)\right]_{l} \leq[T: B]_{l}<\infty$ (Prop. 1 (c)). And so, repeating the above argument, we obtain $[T: B]_{r} \leq\left[T^{\prime}: B\right]_{r}$ $<\infty$, which means $A / B$ is right locally finite. We have proved thus $(\mathrm{A}) \Rightarrow(\mathrm{B})$ $\Longrightarrow\left(\mathrm{C}^{\prime}\right) \Longrightarrow\left(\mathrm{A}^{\prime}\right)$, and symmetrically $\left(\mathrm{A}^{\prime}\right) \Rightarrow\left(\mathrm{B}^{\prime}\right) \Longrightarrow(\mathrm{C}) \Longrightarrow(\mathrm{A})$.

Corollary 2. Let $A$ be left locally finite over a regular subring $B$. If the condition (1) is satisfied, then ( $H$ is simple and) $A$ is h-Galois and locally finite over $H$. And, if $A / B$ is Galois and the condition (1) is satisfied then $A / B$ is $h$ Galois, and conversely.

Proof. Let $B^{\prime}$ be an arbitrary intermediate ring of $A / B[E]$ with $\left[B^{\prime}: B\right]_{l}$ $<\infty$. Then, by Prop. 1 (c), we have $\left[V: V_{A}\left(B^{\prime}\right)\right]_{l} \leq\left[B^{\prime}: B\right]_{l}<\infty$. Since $A$ is $B \cdot V$-A-irreducible (Th. 1), $A$ is $V \cdot H$-A-irreducible much more and $H$ is simple by Prop. 1 (a). And then, by Prop. 1 (b), it follows $\left[V_{A}^{2}\left(B^{\prime}\right): H\right]_{r}$ $\leq\left[V: V_{A}\left(B^{\prime}\right)\right]_{l}<\infty$, which proves evidently the right local finiteness of $A / H$. Hence, Prop. 4 asserts that $A / H$ is locally finite and $h$-Galois. The latter assertion is a direct consequence of Th. 1 and Prop. 4.

The following theorem coincides essentially with [10, Th. 3].

Theorem 2. Let $A$ be left locally finite over a regular subring $B$, and the condition (1) satisfied. If $A^{\prime}$ is a simple intermediate ring of $A / H$ with $\left[A^{\prime}: H\right]_{l}$ $<\infty$, then $A^{\prime}$ is f-regular and $V_{A}^{2}\left(A^{\prime}\right)=A^{\prime}$. 
Proof. By Cor. 2, $A / H$ is $h$-Galois and locally finite. If $A_{0}$ is an arbitrary intermediate ring of $A / A^{\prime}[E]$ with $\left[A_{0}: H\right]_{l}<\infty$ then $A$ is $A_{0}-A$-irreducible and $A-V \cdot H$-irreducible (Prop. 4). Hence, $\left[A_{0}: H\right]_{l} \geq\left[V: V_{A}\left(A_{0}\right)\right]_{r} \geq\left[V_{A}^{2}\left(A_{0}\right)\right.$ : $H]_{l} \geq\left[A_{0}: H\right]_{l}$ by Prop. 1 (b), whence it follows $\left[A_{0}: H\right]_{l}=\left[V: V_{A}\left(A_{0}\right)\right]_{r}$. And then, Prop. 3 (a) asserts that $\operatorname{Hom}_{H_{l}}\left(A_{0}, A\right)=\left(\tilde{V} \mid A_{0}\right) A_{r}$, which means that $\tilde{V} A$, is dense in $\operatorname{Hom}_{H_{l}}(A, A)$. And then, the proof of $[10, \mathrm{Th} .3]$ asserts that $A^{\prime}$ is regular. Accordingly, $\left[V: V_{A}\left(A^{\prime}\right)\right]_{r} \leq\left[A^{\prime}: H\right]_{l}<\infty$ by Th. 1 and Prop. 1 (b), and $V_{A}^{2}\left(A^{\prime}\right)=J\left(\widetilde{V}\left(A^{\prime}\right), A\right)=A^{\prime}$ by Cor. 1 .

Lemma 4. Let $A / B$ be left locally finite, and the condition (1) satisfied. If $\rho$ is a $B$-ring homomorphism of an intermediate ring $A_{1}$ of $A / B$ with $\left[A_{1}: B\right]_{l}$ $<\infty$ onto a simple intermediate ring $A_{2}$ of $A / B$ such that $V_{A}\left(A_{2}\right)$ is a division ring, then $\rho$ is contained in $\mathbb{B}\left(A_{0}, A / B\right) \mid A_{1}$ for any regular intermediate ring $A_{0}$ of $A / A_{1}$ with $\left[A_{0}: B\right]_{l}<\infty$.

Proof. Let $\mathfrak{\mathscr { S }}=\mathbb{B}\left(A_{0}, A / B\right)$. Since $\left[A_{2}: B\right]_{l} \leq\left[A_{1}: B\right]_{l}<\infty$ and $V_{A}\left(A_{2}\right)$ is a division ring, $A$ is $A_{2}-A$-irreducible (Th. 1). And, we have $\operatorname{Hom}_{B_{l}}\left(A_{1}, A\right)=$ $\left(\mathscr{S} \mid A_{1}\right) A_{r}=\sum_{1}^{s}\left(\sigma_{i} \mid A_{1}\right) A_{r}$ with some $\sigma_{i} \in \mathfrak{S}$, for $\left[\operatorname{Hom}_{B_{l}}\left(A_{1}, A\right): A_{r}\right]_{r}=$ $\left[A_{1}: B\right]_{l}<\infty$. Now, the rest of the proof proceeds in the same way as in the proof of [4, Lemma 3.11].

Theorem 3. Let $A / B$ be left locally finite, and the conditions (1), (2) satisfied. If $B_{1} \supseteq B_{2}$ are regular intermediate rings of $A / B$ with $\left[B_{1}: B\right]_{l}<\infty$ then $\leftrightarrow\left(B_{2}\right.$, $A / B)=\mathbb{B}\left(B_{1}, A / B\right) \mid B_{2}$.

Proof. Let $\sigma$ be an arbitrary element of $\$\left(B_{2}, A / B\right)$, and $B_{3}=B_{2} \sigma$. We set $V_{i}=V_{A}\left(B_{i}\right)=\sum_{1}^{m_{i}} U_{i} g_{p q}^{(i)}(i=2,3)$, where $\left\{g_{p q}^{(i)}\right.$ 's $\}$ is a system of matrix units and $U_{i}=V_{V_{i}}\left(\left\{g_{p g}{ }^{(i)}\right.\right.$ 's $\left.\}\right)$ is a division ring. If $m_{2} \geq m_{3}$ then we can consider the subrings $A_{2}, A_{3}$ of $A$ defined as follows:

$$
\begin{aligned}
& A_{2}=\sum_{1}^{m_{3}} B_{2} g_{p y}^{(2)}+B_{2} g, \text { where } g=\sum_{m_{3}+1}^{m_{2}} g_{p p}^{(2)}, \text { and } \\
& A_{3}=\sum_{1}^{m_{3}} B_{3} g_{p q}^{(3)} .
\end{aligned}
$$

Evidently, $A_{2}$ is an intermediate ring of $A / B_{2}$ with $\left[A_{2}: B\right]_{l}<\infty, A_{3}$ a simple intermediate ring of $A / B_{3}$, and $V_{A}\left(A_{3}\right)=U_{3}$ a division ring. As $\left\{g_{p q}^{(i)} \mathrm{s}\right\}$ is linearly independent over $B_{i}$, we can define a $B$-linear map $\rho$ of $A_{2}$ onto $A_{3}$ by the following rule: 


$$
\left\{\begin{array}{l}
\left(B_{2} g\right) \rho=0, \\
\left(\sum_{1}^{m_{3}} b_{p q}^{(2)} g_{p q}^{(2)}\right) \rho=\sum_{1}^{m_{3}}\left(b_{p q}^{(2)} \sigma\right) g_{p q}^{(3)} \quad\left(b_{p q}^{(2)} \in B_{2}\right) .
\end{array}\right.
$$

Then, one will easily see that $\rho$ is a ring homomorphism and $\sigma=\rho \mid B_{2}$. If $A_{0}$ is an arbitrary regular intermediate ring of $A / A_{2}\left[B_{1}\right]$ with $\left[A_{0}: B\right]_{l}<\propto$ then $\rho$ is contained in $\mathbb{B}\left(A_{0}, A / B\right) \mid A_{2}$ (Lemma 4), so that $\sigma=\rho\left|B_{2} \in \mathbb{B}\left(A_{0}, A / B\right)\right| B_{2}$ $=\left(\mathscr{S}\left(A_{0}, A / B\right) \mid B_{1}\right)\left|B_{2} \subseteq \mathbb{S}\left(B_{1}, A / B\right)\right| B_{2}$ by (2). On the other hand, if $m_{2} \leq m_{3}$ then the same argument applied to $\sigma^{-1}$ (instead of $\sigma$ ) enables us to find a simple intermediate ring $A_{0}$ of $A / B_{3}$ with $\left[A_{0}: B\right]_{l}<\propto$ such that $V_{A}\left(A_{0}\right)$ is a division ring and $\sigma^{-1}=\rho \mid B_{3}$ for some $\rho \in \mathbb{B}\left(A_{0}, A / B\right)$. Applying again the above argument to $\rho^{-1}$, we can find a simple intermediate ring $A^{*}$ of $A /\left(A_{0} \rho\right)$ $\left[B_{1}\right]$ with $\left[A^{*}: B\right]_{l}<\infty$ such that $V_{A}\left(A^{*}\right)$ is a division ring and $\rho^{-1}=\tau \mid A_{0} \rho$ for some $\tau \in \mathbb{B}\left(A^{*}, A / B\right)$. Then, $\sigma=\rho^{-1}\left|B_{2}=\tau\right| B_{2} \in \mathbb{B}\left(A^{*}, A / B\right) \mid B_{2} \subseteq \mathbb{B}\left(B_{1}\right.$, $A / B) \mid B_{2}$. Hence, in either cases, we have seen $\mathbb{B}\left(B_{2}, A / B\right) \subseteq \&\left(B_{1}, A / B\right) \mid B_{2}$, whence it follows eventually $\$\left(B_{2}, A / B\right)=\$\left(B_{1}, A / B\right) \mid B_{2}$.

Corollary 3. Let $A$ be left locally finite over a regular subring $B$, and $\mathfrak{H}$ an automorphism group of $A$ containing $\widetilde{V}$. If $\mathfrak{S}_{\alpha} A_{r}$ is dense in $\operatorname{Hom}_{B_{1}}(A, A)$ then $\mathbb{B}\left(B^{\prime}, A / B\right)=\mathfrak{S}_{\mid} B^{\prime}$ for each regular intermediate ring $B^{\prime}$ of $A / B$ with $\left[B^{\prime}: B\right]_{l}<\infty$. In particular, if $A / B$ is $h$-Galois and left locally finite, then the condition (2) is fulfilled. (Cf. [4, Cor. 3.7].)

Proof. If $B_{0}=B^{\prime}[E]$, then $\mathbb{B}\left(B_{0}, A / B\right) \subseteq \operatorname{Hom}_{B_{l}}\left(B_{0}, A\right)=\left(\$_{2} \mid B_{0}\right) A_{r}$, whence it follows $(B)\left(B_{0}, A / B\right)=\mathfrak{F} \mid B_{0}$ (Lemma 2 (c)). Now, the same argument as in the proof of Th. 3 enables us to see that $\mathbb{B}\left(B^{\prime}, A / B\right) \subseteq \mathbb{S}\left(B_{0}, A / B\right)\left|B^{\prime}=\mathfrak{g}\right| B^{\prime}$, whence it follows $\mathbb{B}\left(B^{\prime}, A / B\right)=\mathfrak{g} \mid B^{\prime}$.

2. $q$-Galois Extensions. $A / B$ is said to be $q$-Galois (resp. right $q$-Galois) if $B$ is regular and the conditions (1), (2) (resp. $\left(1^{\prime}\right),\left(2^{\prime}\right)$ ) are satisfied. To be easily verified, if $A$ is a division ring, the notion of $q$-Galois coincides with that of quasi-Galois defined in [8] provided $A / B$ is left locally finite (cf. [8] and Remark 2). And, $A / B$ is said to be locally $h$-Galois if for each finite subset $F$ of $A$ there exists such an intermediate ring $A^{\prime}$ of $A / B[F]$ that $A^{\prime} / B$ is $h$ Galois. Needless to say, if $A / B$ is $h$-Galois or locally Galois then it is locally $h$-Galois.

Propositron 5. If $A / B$ is locally $h$-Galois and left locally finite then it is $q$ Galoiș. 
Proof. Let $B_{1} \supseteq B_{2}$ be regular intermediate rings of $A / B$ with $\left[B_{1}: B\right]_{l}<\infty$, and $\sigma$ an arbitrary element of $\mathbb{S}\left(B_{1}, A / B\right)$. Then, the simple rings $V_{A}\left(B_{1}\right)$, $V_{A}\left(B_{2}\right)$ and $V_{A}\left(B_{1} \sigma\right)$ are represented as the complete matrix rings over division rings with the systems of matrix units $\Gamma_{1}, \Gamma_{2}$ and $\Gamma_{3}$, respectively. Now, for an arbitrary finite subset $F$ of $A$, choose an intermediate ring $A^{*}$ of $A / B_{1}\left[B_{1} \sigma\right.$, $\left.F, E, \Gamma_{1}, \Gamma_{2}, \Gamma_{3}\right]$ such that $A^{*} / B$ is $h$-Galois. Then, by Cor. $3, \sigma$ can be extended to an automorphism $\sigma^{*}$ of $A^{*}$. Since $V_{A^{*}}(B)$ and $V_{A^{*}}\left(B_{2} \sigma\right)=V_{A^{*}}\left(B_{2}\right) \sigma^{*}$ are simple rings, they are the complete matrix rings over division rings with the systems of matrix units $\Gamma^{*}$ and $\Gamma_{2}^{*}$, respectively. If we set $B^{*}=B_{2}\left[B_{2} \sigma\right.$, $\left.F, E, \Gamma^{*}, \Gamma_{2}^{*}\right]\left(\subseteq A^{*}\right), B^{*}$ is a regular subring of $A$ left finite over $B$ such that $V_{B^{*}}(B)$ and $V_{B^{*}}\left(B_{2} \sigma\right)$ are simple. Hence, we have seen that there exists a directed set $\left\{B_{\lambda}^{*}\right\}$ of regular intermediate rings $B_{\lambda}^{*}$ of $A / B_{2}\left[B_{z} \sigma\right]$ such that $\left[B_{\lambda}^{*}: B\right]_{l}<\infty, A=\cup B_{\lambda}^{*}$ and that $V_{B_{\lambda}^{*}}(B)$ and $V_{B_{\lambda}^{*}}\left(B_{2} \sigma\right)$ are simple. It follows therefore $V=\cup V_{B_{\lambda}^{*}}(B)$ and $V_{A}\left(B_{2} \sigma\right)=\cup V_{B_{\lambda}^{*}}\left(B_{2} \sigma\right)$ are simple by [4, Lemma 1.1], which proves (2). Moreover, noting that $B^{*}$ contains $E$, we see that $\operatorname{Hom}_{B l}\left(B^{*}, A\right)=\operatorname{Hom}_{\rho_{l}}\left(B^{*}, A^{*}\right) A_{r}=\left(\left(\&\left(A^{*} / B\right) \mid B^{*}\right) A_{r}^{*}\right) A_{r} \subseteq \&\left(B^{*}, A / B\right) A_{r}$. And so, by (2), it follows eventually $\operatorname{Hom}_{\imath}\left(B_{2}, A\right)=\operatorname{Hom}_{B_{l}}\left(B^{*}, A\right) \mid B_{2}=\left(\$\left(B^{*}\right.\right.$, $\left.A / B) \mid B_{2}\right) A_{r}=\mathbb{B}\left(B_{2}, A / B\right) A_{r}$.

We insert here [4, Th. 2.3] as an easy consequence of Cors. 2 and 3.

Proposition 6. If $A / B$ is Galois and locally Galois then $A / B$ is BS-locally Galois, and conversely.

Proof. $A / B$ is $h$-Galois by Cor. 2, so that for each shade $B^{\prime}$ we have $\mathbb{B}\left(B^{\prime} / B\right) \subseteq \mathbb{S}\left(B^{\prime}, A / B\right)=\mathbb{S} \mid B^{\prime}$ (Cor. 3). And the converse part is obvious.

By the validity of Th. 1, the proof of the next lemma proceeds just like that of $[5$, Lemma 8$]$ did.

Lemma 5. Let $A / B$ be left locally finite, the condition (1) satisfied, and $A^{*}$ a regular subring of $A$ containing $B$. If $F$ is an arbitrary finite subset of $A^{*}$, then $A^{*}$ contains a regular subring $B^{\prime}$ of $A$ such that $B^{\prime} \supseteq B[F]$ and $\left[B^{\prime}: B\right]_{l}<\infty$.

Lemma 6. Let $A / B$ be $q$-Galois and left locally finite. If $A^{\prime}$ is an f-regular intermediate ring of $A / B$ then $\left(H \cap A^{\prime}\right) \$\left(A^{\prime}, A / B\right) \subseteq H$.

Proof. Let $\sigma$ be an arbitrary element of $\$\left(A^{\prime}, A / B\right)$, and $h$ an arbitrary one of $H \cap A^{\prime}$. And, choose a simple intermediate ring $B^{\prime}$ of $A^{\prime} / B[h]$ such 
that $V_{A}\left(B^{\prime}\right)=V_{A}\left(A^{\prime}\right)$ and $\left[B^{\prime}: B\right]_{l}<\infty$. Then, by Lemma 5 the regular subring $A^{\prime} \sigma$ contains a simple subring $B^{*}$ containing $B^{\prime} \sigma$ such that $V_{A}\left(B^{*}\right)$ is simple and $\left[B^{*}: B\right]_{l}<\infty$. Here, needless to say, $B^{\prime \prime}=B^{*} \sigma^{-1}$ is a regular subring of $A$ as an intermediate ring of $A^{\prime} / B^{\prime}$. And so, $\tau^{\prime \prime}=\sigma^{-1} \mid B^{*}$ is contained in $B\left(B^{*}, A / B\right)$. If $v$ is an arbitrary element of $V, \tau^{\prime \prime}=\tau \mid B^{*}$ with some $\tau \in$ $\mathbb{B}\left(B^{*}[E, v], A / B\right)$ (Th. 3). As $v \tau$ is contained in $V$, we have $h \cdot v \tau=v \tau \cdot h$, whence it follows $h \sigma \cdot v=v \cdot h \sigma$. We see therefore $h \sigma \in H$.

Now, we can prove the following theorem that corresponds to [8, Cor. 1$]$.

Theorem 4. If $A$ is $q$-Galois and left locally finite over $B$, then $H / B$ is outer Galois and $\mathbb{B}(H, A / B)=\mathbb{B}(H / B)$.

Proof. Let $B^{\prime}$ be an arbitrary intermediate ring of $H / B[\Delta]$ with $\left[B^{\prime}: B\right]_{l}$ $<\infty$ (Cor. 2). Since $B^{\prime}\left(B^{\prime}, A / B\right) \subseteq H$ (Lemma 6), Lemma 2 (a) yields $\left[\&\left(B^{\prime}, A / B\right) H_{r}: H_{r}\right]_{r} \leq\left[B^{\prime}: B\right]_{l}<\infty$. Hence, $\mathbb{B}\left(B^{\prime}, A / B\right) H_{r}=\oplus_{1}^{t} \sigma_{i} H_{r}$ with some $\sigma_{i} \in \mathbb{B}\left(B^{\prime}, A / B\right)$ and so $\mathbb{B}\left(B^{\prime}, A / B\right)=\mathbb{B}\left(B^{\prime}, H / B\right)=\left\{\sigma_{1}, \ldots, \sigma_{t}\right\}$ by Lemma 2 (c). Now, we set $H=\cup B_{\alpha}$, where $B_{\alpha}$ ranges over all the intermediate rings of $H / B[\Delta]$ with $\left[B_{\alpha}: B\right]_{l}<\infty$. We can consider then the inverse limit $\mathscr{Y}=\lim \mathbb{S}\left(B_{\alpha}, A / B\right)$, that may be regarded as a set of $B$-(ring) isomorphisms of $H$ into $H$. Since every $\mathbb{B}\left(B_{\alpha}, A / B\right)$ is finite and $\mathbb{B}\left(B_{\alpha}, A / B\right) \mid B_{\beta}=$

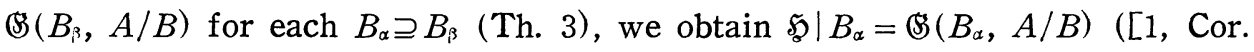
3.9]). If $T$ is an arbitrary subring of $H$ properly containing $B$ with $[T: B]_{t}$ $<\infty$ then there exists some $B_{\alpha}$ containing $T$ and then $J\left(B\left(B_{\alpha}, A / B\right), B_{\alpha}\right)=B$ by Remark 1. Combining this with $\mathfrak{g} \mid B_{\alpha}=\mathbb{B}\left(B_{\alpha}, A / B\right)$, we readily see that $J\left(\mathfrak{g}_{2}, H\right)=B$. Further, if $\sigma$ is in $\mathscr{S}$ then for each $B_{\alpha}$ we can find a positive integer $n_{\alpha}$ such that $\sigma^{n_{\alpha}} \mid B_{\alpha}=1$, which proves $H_{\sigma}=H$, that is, $\sigma$ is an automorphism of $H$. Finally, if $\tau$ is an arbitrary element of $(B(H, A / B)$ then $H \tau \subseteq H$ (Lemma 6), and so we obtain $\$(H, A / B)=\$(H / B)$ by Prop. 2 (a).

Corollary 4. Let $A / B$ be $q$-Galois and left locally finite. If $A^{\prime}$ is a simple intermediate ring of $A / H$ with $\left[A^{\prime}: H\right]_{l}<\infty$ then $A^{\prime}$ is f-regular and $\mathbb{B}\left(A^{\prime}\right.$, $A / B) \mid H \subseteq(B(H / B)$.

Proof. The first assertion is contained in Th. 2, and then $H \&\left(A^{\prime}, A / B\right) \subseteq$ $H$ (Lemma 6). Recalling now that $H / B$ is outer Galois (Th. 4), the latter is obvious by Prop. 2 (a). 
3. $h-q$-Galois Extensions. $A / B$ is said to be $h-q$-Galois (resp. right $h-q$ Galois) if $B$ is regular and $A / B^{\prime}$ is $q$-Galois (resp. right $q$-Galois) for each regular intermediate ring $B^{\prime}$ of $A / B$ with $\left[B^{\prime}: B\right]_{l}<\infty\left(\operatorname{resp} .\left[B^{\prime}: B\right]_{r}<\infty\right)$. If $A / B$ is left locally finite and locally $h$-Gaiois then it is $h-q$-Galois by Prop. 5 and Cor. 1. Moreover, in case $A$ is a division ring, the notion of $q$-Galois coincides with that of $h$-q-Galois (Lemma 2 ).

Now, assume that $A / B$ is $h-q$-Galois and left locally finite. If $B^{\prime}$ is a regular intermediate ring of $A / B$ with $\left[B^{\prime}: B\right]_{l}<\infty$, then $A / B^{\prime}$ is $q$-Galois and $V_{A}^{2}\left(B^{\prime}\right) / B^{\prime}$ is outer Galois (Th. 4), and so $H\left[B^{\prime}\right]$ is a simple ring (Prop. 2). Recalling that $A / H$ is locally finite (Cor. 2), Th. 2 yields $H\left[B^{\prime}\right]=V_{A}^{2}\left(B^{\prime}\right)$. (This fact will be used often without mention in the sequel.) Since $B\left(V_{A}^{2}\left(B^{\prime}\right) /\right.$ $\left.B^{\prime}\right) \mid H \subseteq(B(H / B)$ (Cor. 4$), \sigma \rightarrow \sigma \mid H$ is a continuous monomorphism of compact $\mathbb{B}\left(V_{A}^{2}\left(B^{\prime}\right) / B^{\prime}\right)$ into $\mathbb{B}\left(H / H \cap B^{\prime}\right)$ and its image is a Galois group of $H / H \cap B^{\prime}$. Hence, we see that $\sigma \rightarrow \sigma \mid H$ is an isomorphism onto $B\left(H / H \cap B^{\prime}\right)$. (Cf. [4] or [9]). By the aid of this fact, the same argument as in the proof of $[5$, Lemma 9] enables us to see that if $A$ is $h-q$-Galois and left locally finite over $B$ and $A^{\prime}$ is a regular intermediate ring of $A / B$ with $\left[H\left[A^{\prime}\right]: H\right]_{l}<\infty$ then $H\left[A^{\prime}\right]$ is outer Galois and locally finite over $A^{\prime}$ and $\mathbb{B}\left(H\left[A^{\prime}\right] / A^{\prime}\right) \approx \mathbb{B}\left(H / H \cap A^{\prime}\right)$ by contraction. Accordingly, by the validity of Lemma 5 , we can apply the same argument as in the proof of $[5, \mathrm{Th} .6]$ to obtain the next theorem that is stated without proof.

Theorem 5. Let $A$ be $h-q$-Galois and left locally finite over $B$. If $A^{\prime}$ is a regular intermediate ring of $A / B$, and $H^{\prime}$ an intermediate ring of $H / B$ that is Galois over $B$, then $H^{\prime}\left[A^{\prime}\right]$ is outer Galois and locally finite over $A^{\prime}$ and $B\left(H^{\prime}\left[A^{\prime}\right] /\right.$ $\left.A^{\prime}\right) \approx \mathbb{S}\left(H^{\prime} / H^{\prime} \cap A^{\prime}\right)$ (algebraically and topologically) by contraction.

As the first corollary to Th. 5 , we shall remark that if $A / B$ is $h-q$-Galois and left locally finite then the condition (2) can be sharpened as follows:

$\left(2^{*}\right) \mathbb{B}\left(A_{1}, A / B\right) \mid A_{2} \subseteq \mathbb{B}\left(A_{2}, A / B\right)$ for each $f$-regular intermediate rings $A_{1} \supseteq A_{2}$ of $A / B$.

To prove $\left(2^{*}\right)$, let $\sigma$ be an arbitrary element of $\&\left(A_{1}, A / B\right)$, and $B_{1}$ a simple intermediate ring of $A_{1} / B$ with $\left[B_{1}: B\right]_{l}<\infty$ and $V_{A}\left(B_{1}\right)=V_{A}\left(A_{1}\right)$. If $B_{2}$ is an arbitrary regular subring of $A$ between $A_{1}$ and $B$ with $\left[B_{2}: B\right]_{l}<\infty$, then we can find a regular subring $B^{*}$ of $A$ between $A_{1} \sigma$ and $\left(B_{1}\left[B_{2}\right]\right)_{\sigma}$ with $\left[B^{*}: B\right]_{l}<\infty$ (Lemma 5). Evidently $B^{\prime}=B^{*} \sigma^{-1}$ is regular as an intermediate 
ring of $A_{1} / B_{1}$. Hence, $\sigma^{\prime}=\sigma \mid B^{\prime}$ is in $\&\left(B^{\prime}, A / B\right)$, and so $B_{2} \sigma=B_{2} \sigma^{\prime}$ is regular by the condition (2). Now, let $B_{2}$ be specialized as a simple intermediate ring of $A_{2} / B$ with $\left[B_{2}: B\right]_{1}<\infty$ and $V_{A}\left(B_{2}\right)=V_{A}\left(A_{2}\right)$. Since $A_{2}=\left(H \cap A_{2}\right)\left[B_{2}\right]$ by Th. 5 and Prop. 2, Lemma 6 yields $V_{A}\left(A_{2} \sigma\right)=V_{A}\left(\left(\left(H \cap A_{2}\right)_{\sigma}\right)\left[B_{2} \sigma\right]\right)=V_{A}\left(B_{2} \sigma\right)$. Hence, $V_{A}\left(B_{2} \sigma\right)$ being simple by the above remark, it follows that $\sigma \mid A_{2}$ is contained in $\$\left(A_{2}, A / B\right)$.

Corollary 5. Let $A / B$ be h-q-Galois and left locally finite. If $B^{\prime}$ is a regular intermediate ring of $A / B$ with $\left[B^{\prime}: B\right]_{l}<\infty$ then $\mathbb{B}\left(B^{\prime}, A / B\right)=\mathbb{B}\left(V_{A}^{2}\left(B^{\prime}\right)\right.$, $A / B) \mid B^{\prime}$.

Proof. By Th. $5, H^{*}=V_{A}^{2}\left(B^{\prime}\right)=H\left[B^{\prime}\right]$ is outer Galois over $B^{\prime}$. We set here $H^{*}=\cup B_{\alpha}^{\prime}$, where $B_{\alpha}^{\prime}$ ranges over all the $\mathbb{S}\left(H^{*} / B^{\prime}\right)$-invariant shades. Now, let $\rho$ be an arbitrary element of $\mathbb{B}\left(B^{\prime}, A / B\right)$. Then, the set $\xi_{\alpha}=\left\{\rho^{\prime} \in\right.$ $\left.\mathbb{B}\left(B_{\alpha}^{\prime}, A / B\right) ; \rho^{\prime} \mid B^{\prime}=\rho\right\}$ is non-empty (Th. 3). If $\rho^{\prime}$ and $\rho^{\prime \prime}$ are in $E_{\alpha}$ then $\rho^{\prime \prime}=\rho^{\prime} \varepsilon$ with some $B^{\prime} \rho$-(ring) isomorphism $\varepsilon$ between regular subrings $B_{\alpha}^{\prime} \rho^{\prime}$ and $B_{\alpha}^{\prime} \rho^{\prime \prime}$. As $B_{\alpha}^{\prime}=\left(H \cap B_{\alpha}^{\prime}\right)\left[B^{\prime}\right]$ (Th. 5 and Prop. 2), $B_{\alpha}^{\prime} \rho^{\prime} \subseteq H\left[B^{\prime} \rho\right]=V_{A}^{2}\left(B^{\prime} \rho\right)$ by Lemma 6. And so, recalling that $A$ is $q$-Galois and left locally finite over $B^{\prime} \rho$ and $B_{\alpha}^{\prime} \rho^{\prime} / B^{\prime} \rho$ is Galois, by [4, Cor. 3.9], Lemma 6 and Prop. 2 (a), we see that $\mathbb{B}\left(B_{\alpha}^{\prime} \rho^{\prime} / B^{\prime} \rho\right)=\mathbb{B}\left(V_{A}^{2}\left(B^{\prime} \rho\right) / B^{\prime} \rho\right) \mid B_{\alpha}^{\prime} \rho^{\prime}=\mathbb{B}\left(B_{\alpha}^{\prime} \rho^{\prime}, A / B^{\prime} \rho\right)$. Consequently, $\mathbb{B}\left(B_{\alpha}^{\prime} \rho^{\prime}, A / B^{\prime} \rho\right)=\mathbb{B}\left(B_{\alpha}^{\prime} \rho^{\prime} / B^{\prime} \rho\right) \approx \mathbb{S}\left(B_{\alpha}^{\prime} / B^{\prime}\right)$ is finite, and so $\xi_{\alpha}$ is finite, too. Thus, by $\left[1\right.$, Th. 3.6], the inverse limit $\mathfrak{F}=\lim \xi_{\alpha}$ is non-empty, which means that $\rho \in \mathbb{B}\left(B^{\prime}, A / B\right)$ can be extended to an isomorphism $\rho^{*}$ of $H^{*}$ into $A$. Since $\left(H \cap B_{\alpha}^{\prime}\right) \rho^{\prime} \subseteq H$ for each $\rho^{\prime} \in \mathfrak{E}_{\alpha}$ (Lemma 6), $H^{*} \rho^{*}=\left(\cup\left(H \cap B_{\alpha}^{\prime}\right)\left[B^{\prime}\right]\right) \rho^{*}$ is to be regular. Hence, we have seen $\mathbb{B}\left(B^{\prime}, A / B\right) \subseteq \mathbb{S}\left(H^{*}, A / B\right) \mid B^{\prime}$. The converse inclusion is secured by $(2 *)$.

Corollary 6. Let $A / B$ be h-q-Galois and left locally finite. If $B^{\prime}$ is a regular intermediate ring of $A / B[\Delta]$ with $\left[B^{\prime}: B\right]_{l}<\infty$ then $H^{*}\left[B^{\prime}\right]=H^{*} \cdot B$ and $\left[H^{*}\left[B^{\prime}\right]: H^{*}\right]_{l}=\left[A^{*}: H \cap A^{*}\right]_{l}=\left[B^{\prime}: H \cap B^{\prime}\right]_{l}$ for each intermediate ring $H^{*}$ of $H / H \cap B^{\prime}$ and each intermediate ring $A^{*}$ of $H\left[B^{\prime}\right] / B^{\prime}$.

Proof. We set $H^{\prime}=H \cap B^{\prime}$ and $\hat{B}^{\prime}=\hat{S}\left(H\left[B^{\prime}\right] / B^{\prime}\right)$. Then, $H^{\prime}$ is simple by Th. 4 and Prop. 2. If $M$ is an arbitrary $\$\left(H / H^{\prime}\right)$-invariant shade then $\mathbb{B}\left(M\left[B^{\prime}\right] / B^{\prime}\right)=\mathbb{S}^{\prime}\left|M\left[B^{\prime}\right] \approx \mathbb{S}^{\prime}\right| M=\mathbb{S}\left(M / H^{\prime}\right)$ (Th. 5), which implies $\left[M\left[B^{\prime}\right]\right.$ : $\left.B^{\prime}\right]=\left[M: H^{\prime}\right]$. Accordingly, we obtain $\left[M\left[B^{\prime}\right]: M\right]_{l}=\left[B^{\prime}: H^{\prime}\right]_{l}$. On the other hand, by the validity of Th. 5 , Lemma 3 applies to obtain $\left[M \cdot B^{\prime}: M\right]_{l}$ 
$=\left[B^{\prime}: H^{\prime}\right]_{l} . \quad$ It follows therefore $M\left[B^{\prime}\right]=M \cdot B^{\prime} . \quad$ Now, it will be easy to see that $H\left[B^{\prime}\right]=H \cdot B^{\prime}=\oplus_{1}^{t} H b_{i}^{\prime}$, where $\left\{b_{i}^{\prime \prime} \mathbf{s}\right\}$ is an arbitrary linearly independent left $H^{\prime}$-basis of $B^{\prime}$. And so, we have $H^{*}\left[B^{\prime}\right]=J\left(B^{\prime}\left(H^{*}\left[B^{\prime}\right]\right), \oplus_{1}^{t} H b_{i}^{\prime}\right)$ $=\oplus_{1}^{t} H^{*} b_{i}^{\prime}$ (Prop. $2(\mathrm{~b})$ ), whence $H^{*}\left[B^{\prime}\right]=H^{*} \cdot B^{\prime}$. And, at the same time, the latter assertion is also obvious by Th. 5 and Prop. 2 (b).

If $A / B$ is $h-q$-Galois and left locally finite, we can prove the following sharpening of Th. 3 , which is at the same time an extension of $[6, \mathrm{Th} .5]$ to simple rings.

Theorem 6. Let $A / B$ be $h-q$-Galois and left locally finite. If $A_{1} \supseteq A_{2}$ are $f$ regular intermediate rings of $A / B$ then $\$\left(A_{2}, A / B\right)=\mathbb{S}\left(A_{1}, A / B\right) \mid A_{2}$.

Proof. (I) We shall prove first our theorem for regular intermediate rings $A_{1} \supseteq A_{2}$ of $A / H$ with $\left[A_{1}: H\right]_{l}<\infty$. By the validity of $\left(2^{*}\right)$, it suffices to prove that $\mathbb{S}\left(A_{2}, A / B\right) \subseteq \mathbb{S}\left(A_{1}, A / B\right) \mid A_{2}$. Choose a simple intermediate ring $B_{2}^{\prime}$ of $A_{2} / B$ with $\left[B_{2}^{\prime}: B\right]_{l}<\infty$ and $V_{A}\left(B_{2}^{\prime}\right)=V_{A}\left(A_{2}\right)$ (Th. 2). And then, between $A_{1}$ and $B_{2}^{\prime}$ there exists a regular subring $B_{1}$ of $A$ with $\left[B_{1}: B\right]_{l}<\infty$ and $A_{1}=V_{A}^{2}\left(B_{1}\right)=H\left[B_{1}\right]$. If $B_{2}=A_{2} \cap B_{1}$ then $B_{2}^{\prime} \subseteq B_{2} \subseteq A_{2}=V_{A}^{2}\left(B_{2}^{\prime}\right)$, and hence $B_{2}$ is a regular subring of $A$ left finite over $B$ (Th. 4 and Prop. 2 (a)) and $A_{2}=V_{A}^{2}\left(B_{2}\right)=H\left[B_{2}\right]$. Since $\mathbb{S}\left(A_{2}, A / B\right)\left|B_{2}=\mathbb{B}\left(B_{2}, A / B\right)=\mathbb{B}\left(B_{1}, A / B\right)\right| B_{2}=$ (B) $\left.A_{1}, A / B\right) \mid B_{2}$ (Cor. 5 and Th. 3), for each $\sigma \in \mathbb{B}\left(A_{2}, A / B\right)$ we can find some $\rho \in \mathbb{S}\left(A_{1}, A / B\right)$ with $\rho\left|B_{2}=\sigma\right| B_{2}$. As $A_{2} \sigma=H\left[B_{2} \sigma\right]=H\left[B_{2} \rho\right]=A_{2} \rho$ (Cor. 4), $\sigma \rho^{-1}$ is contained in $\mathbb{S}\left(A_{2} / B_{2}\right)=\mathbb{S}\left(A_{2} / A_{2} \cap B_{1}\right)=\mathbb{S}\left(A_{1} / B_{1}\right) \mid A_{2}$ (Th. 5). Hence, $\sigma$ is in $\&\left(A_{1}, A / B\right) \mid A_{2}$.

(II) Now, assume that $A_{i}$ be $f$-regular, and take simple intermediate rings $B_{i}$ of $A_{i} / B$ with $\left[B_{i}: B\right]_{l}<\infty$ and $V_{A}\left(B_{i}\right)=V_{A}\left(A_{i}\right)(i=1,2)$. Then, $A_{i}^{\prime}=$ $V_{A}^{2}\left(B_{i}\right)=H\left[B_{i}\right]$ are finite over $H$ (Cor. 2), $A_{1}^{\prime} \supseteq A_{2}^{\prime} \supseteq H$ and $A_{i}^{\prime} \supseteq A_{i} \supseteq B_{i}$. Now, let $\sigma_{i}$ be arbitrary elements of $\hat{B}\left(A_{i}, A / B\right)$. Then, by Cor. 5 and $\left(2^{*}\right), \sigma_{i} \mid B_{i}$ $=\tau_{i} \mid B_{i}$ for some $\tau_{i} \in \mathbb{B}\left(A_{i}^{\prime}, A / B\right)$. Recalling that $A_{i}=\left(H \cap A_{i}\right)\left[B_{i}\right]$ (Th. 5 and Prop. 2), we see that $A_{i} \sigma_{i}=\left(\left(H \cap A_{i}\right)_{\sigma_{i}}\right)\left[B_{i} \sigma_{i}\right] \subseteq H\left[B_{i} \tau_{i}\right]=A_{i}^{\prime} \tau_{i}$ (Lemma 6). And so, $\sigma_{i} \tau_{i}^{-1}$ is contained in $\$\left(A_{i}^{\prime} / B_{i}\right) \mid A_{i}$ (Th. 4 and Prop. 2 (a)), whence it follows $\sigma_{i} \in \mathbb{S}\left(A_{i}^{\prime}, A / B\right) \mid A_{i}$. Combining this with $\left(2^{*}\right)$, we obtain $\mathbb{B}\left(A_{i}, A / B\right)$ $=\mathbb{S}\left(A_{i}^{\prime}, A / B\right) \mid A_{i} . \quad$ On the other hand, there holds $\mathbb{\$}\left(A_{2}^{\prime}, A / B\right)=\mathbb{B}\left(A_{1}^{\prime}\right.$, $A / B) \mid A_{2}^{\prime}$ by (I). Hence, it follows $\mathbb{B}\left(A_{2}, A / B\right)=\mathbb{B}\left(A_{2}^{\prime}, A / B\right) \mid A_{2}=\left(\mathbb{B}\left(A_{1}^{\prime}\right.\right.$, $\left.A / B) \mid A_{2}^{\prime}\right)\left|A_{2}=\left(\$\left(A_{1}^{\prime}, A / B\right) \mid A_{1}\right)\right| A_{2}=\mathbb{B}\left(A_{1}, A / B\right) \mid A_{2}$, completing the proof. 
Remark 2. Let $A$ be a division ring, and left locally finite over $B$. Then, $\mathbb{B}\left(B^{\prime}, A / B\right)$ is nothing but the set of all $B$-ring isomorphisms of $B^{\prime}$ into $A$, and the condition (2) is superfluous. Following [6] and [8], we consider the following conditions:

$\left(1^{\circ}\right) \mathbb{S}\left(B^{\prime}, A / B\right) \neq 1$ for each subring $B^{\prime}$ of $A$ properly containing $B$ with $\left[B^{\prime}: B\right]_{l}<\infty$, and $\mathbb{S}\left(B_{1}, A / B\right) \mid B_{2}=\$\left(B_{2}, A / B\right)$ for each intermediate rings $B_{1} \supseteq B_{2}$ of $A / B$ with $\left[B_{1}: B\right]_{l}<\infty$.

$\left(2^{\circ}\right) H / B$ is Galois, and $\left(B\left(B_{1}, A / B\right) \mid B_{2}=\mathbb{B}\left(B_{2}, A / B\right)\right.$ for each intermediate rings $B_{1} \supseteq B_{2}$ of $A / B$ with $\left[B_{1}: B\right]_{l}<\infty$.

$\left(3^{\circ}\right) H / B$ is Galois, and $\mathbb{B}\left(A_{1}, A / B\right) \mid A_{2}=\mathbb{B}\left(A_{2}, A / B\right)$ for each intermediate rings $A_{1} \supseteq A_{2}$ of $A / H$ with $\left[A_{1}: H\right]_{l}<\infty$.

$\left(4^{\circ}\right) J\left(B\left(B^{\prime}, A / B\right), B^{\prime}\right)=B$ for each intermediate ring $B^{\prime}$ of $A / B$ with $\left[B^{\prime}: B\right]_{l}<\infty$.

If $A / B$ is $q$-Galois (and necessarily $h-q$-Galois by Lemma 2 ), then all the conditions $\left(1^{\circ}\right)-\left(4^{\circ}\right)$ are fulfilled by Remark 1 and Ths. 4, 6. Conversely, if $\left(4^{\circ}\right)$ is satisfied then $A / B$ is $q$-Galois. To see this, it will suffice to prove that if $\left\{x_{1}, \ldots, x_{n}\right\}$ is a subset of $B^{\prime}$ that is linearly left independent over $B$ then there exists an element $\xi \in \mathbb{S}\left(B^{\prime}, A / B\right) A_{r}$ such that $x_{i} \xi=0$ for all $i \neq n$ and $x_{n} \xi \neq 0$, where $B^{\prime}$ is an arbitrary intermediate ring of $A / B$ with $\left[B^{\prime}: B\right]_{l}$ $<\infty$. If $n=2$, by $\left(4^{\circ}\right)$ there exists some $\rho \in\left(\dot{S}\left(B^{\prime}, A / B\right)\right.$ with $\left(x_{1} x_{2}^{-1}\right) \rho \neq x_{1} x_{2}^{-1}$, and then one will easily see that $\xi=\rho-1\left(x_{1}^{-1} \cdot x_{1} \rho\right)_{r}$ is an element requested. Now, assume that we can find $\xi_{1}, \ldots, \xi_{n-1} \in \mathbb{S}\left(B^{\prime}, A / B\right) A_{r}$ such that $x_{i} \xi_{j}=$ $\delta_{i j} x_{i}(i, j=1, \ldots, n-1)$. There holds then $x_{i}\left(\sum \xi_{j}-1\right)=0$ for $i=1, \ldots$, $n-1$. If $x_{n}\left(\sum \xi_{j}-1\right) \neq 0$, our assertion is true for $\xi=\sum \xi_{j}-1$. If otherwise $x_{n}=\sum_{1}^{n-1} x_{n} \xi_{j}$ then, say, $\left\{x_{1}, x_{n} \xi_{1}\right\}$ is linearly left independent over $B$. We set here $\xi_{1}=\sum_{1}^{k} \rho_{p} a_{p r}$ with $\rho_{p} \in \mathbb{B}\left(B^{\prime}, A / B\right)$ and $a_{p} \in A$. If $B^{\prime \prime}=B^{\prime}\left[\cup B^{\prime} \mu_{p}\right.$, $\left.\left\{a_{p}{ }^{\prime} \mathrm{s}\right\}\right]$, then by the case $n=2$ there exists an element $\xi^{\prime} \in \mathbb{S}\left(B^{\prime \prime}, A / B\right) A_{r}$ such that $x_{1} \xi^{\prime}=0$ and $x_{n} \xi_{1} \xi^{\prime} \neq 0$. Now, it will be easy to see that $x_{i} \xi_{1} \xi^{\prime}=0$ for $i=$ $1, \ldots, n-1$, so that $\xi=\xi_{1} \xi^{\prime}$ contained in $\mathbb{S}\left(B^{\prime}, A / B\right) A_{r}$ is an element requested.

Next, we shall prove the implications $\left(2^{\circ}\right) \Longrightarrow\left(4^{\circ}\right)$ and $\left(3^{\circ}\right) \Longrightarrow\left(4^{\circ}\right)$. In any rate, we have $J\left(\mathbb{S}^{(}\left(B^{\prime}, A / B\right), B^{\prime}\right) \subseteq J\left(\widetilde{V} \mid B^{\prime}, B^{\prime}\right)=H \cap B^{\prime}$. If $\left(2^{\circ}\right)$ is satisfied then $\mathbb{B}(H / B)\left|H \cap B^{\prime} \subseteq \&\left(B^{\prime}, A / B\right)\right| H \cap B^{\prime}$, whence it follows $J\left(\&\left(B^{\prime}, A / B\right), B^{\prime}\right)$ $=B$. On the other hand, if $\left(3^{\circ}\right)$ is satisfied then $\mathbb{S}(H / B) \subseteq \mathbb{S}\left(H\left[B^{\prime}\right], A / B\right) \mid H$ 


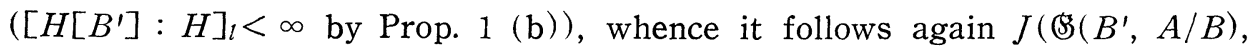
$\left.B^{\prime}\right)=B$.

Since the implication $\left(1^{\circ}\right) \Rightarrow\left(4^{\circ}\right)$ is obvious, we have proved that $A$ is $q$ Galois if and only if any of the equivalent conditions $\left(1^{\circ}\right)-\left(4^{\circ}\right)$ is satisfied (cf. [6, Th. 1] and [8, Th. 3]).

In case $A / B$ is an algebraic field extension, it is well-known that $A / B$ is Galois (in our sense) if and only if it is normal and separable. The next theorem may be regarded as an extension of this fact to simple rings, and contains [6, Cor. 3] as well as [4, Th. 3.5].

Theorem 7. If $A$ is $h-q$-Galois and left locally finite over $B$ and $[A: H]$, $\leq \aleph_{0}$, then $A / B$ is $h$-Galois and $\mathbb{B}\left(A^{\prime}, A / B\right)=\mathbb{B} \mid A^{\prime}$ for each f-regular intermediate ring $A^{\prime}$ of $A / B$. In particular, if $A$ is locally Galois over $B$ and $[A$ : $H]_{l} \leq \aleph_{0}$ then $A / B$ is (8-locally Galois.

Proof. Since $A^{\prime}$ is $f$-regular, we can find an intermediate ring $A^{\prime \prime}$ of $A / H\left[E, A^{\prime}\right]$ with $\left[A^{\prime \prime}: H\right]_{l}<\infty$ (Cor. 2). Now, by the validity of Cors. 2, 4 and Th. 6, we can apply the same argument as in the proof of [4, Lemma 3.9] to see that $\mathbb{B}\left(A^{\prime \prime}, A / B\right)=\mathbb{B} \mid A^{\prime \prime}$. Then, we obtain $\mathbb{B}\left|A^{\prime}=\mathbb{B}\left(A^{\prime \prime}, A / B\right)\right| A^{\prime}=$ $\mathbb{B}\left(A^{\prime}, A / B\right)$ (Th. 6), and in particular $\mathbb{S} \mid H=\mathbb{B}(H, A / B)=\mathbb{B}(H / B)$ (Th. 4). Hence, there holds $J(\mathbb{B}, A)=J(\$ \mid H, H)=B$. And so, $A$ being $B \cdot V-A$ irreducible (Th. 1), $A / B$ is $h$-Galois by Prop. 4. The latter assertion is [4, Th. 4.4] itself, and is clear by the former and Prop. 6.

Next, we shall prove an extension of the latter half of [2, Th. 1], that contains completely [6, Cor. 2].

Theorem 8. Let $A / B$ be h-q-Galois and left locally finite. If $B^{\prime}$ is a regular intermediate ring of $A / B$ with $\left[B^{\prime}: B\right]_{l}<\infty$ then $\infty>\left[B^{\prime}: B\right]^{4)} \geq\left[V: V_{A}\left(B^{\prime}\right)\right]$ $=\left[V_{A}^{2}\left(B^{\prime}\right): H\right]=\left[B^{\prime}: H \cap B^{\prime}\right]$, and in particular $A / B$ is (two-sided) locally finite.

Proof. We set $V_{A}^{2}\left(B^{\prime}\right)=\sum K^{\prime} d_{h^{\prime} k^{\prime}}^{\prime}$, where $\Delta^{\prime}=\left\{d_{h^{\prime} k^{\prime}}^{\prime}\right.$ s $\}$ is a system of matrix units and $K^{\prime}=V_{V^{2} A\left(B^{\prime}\right)}\left(\Delta^{\prime}\right)$ is a division ring (Cor. 2), and consider $T$ $=B^{\prime}\left[E, \Delta, \Delta^{\prime}\right]$ and $H^{\prime}=H \cap T$ (simple by Th. 4 and Prop. 2). Since $H \&\left(V_{A}^{2}\right.$ $(T) / T)=H$ (Cor. 4 ) and $A$ is $B \cdot V$-A-irreducible (Th. 1), Prop. 1 and Lemma

4) In case $\left[B^{\prime}: B\right]_{l}$ coincides with $\left[B^{\prime}: B\right]_{r}$, the equal dimensions will be denoted as $\left[B^{\prime}: B\right]$. 
3 yield $\infty>\left[T: H^{\prime}\right]_{l} \geq\left[V: V_{A}(T)\right]_{l} \geq\left[V_{A}^{2}(T): H\right]_{r} \geq[T \cdot H: H]_{r}=\left[T: H^{\prime}\right]_{r}$. And then, $A$ being $A-V \cdot H$-irreducible by Cor. 2 and Prop. 4, we obtain $[T$ : $\left.H^{\prime}\right]_{r} \geq\left[V: V_{A}(T)\right]_{r} \geq\left[V_{A}^{2}(T): H\right]_{l} \geq[H \cdot T: H]_{l}=\left[T: H^{\prime}\right]_{l}$ again by Prop. 1 and Lemma 3. Hence, it follows $\left[T: H^{\prime}\right]=\left[V: V_{A}(T)\right]=\left[V_{A}^{2}(T): H\right]$ and $[T: B]_{l}=\left[T: H^{\prime}\right]_{l} \cdot\left[H^{\prime}: B\right]_{l}=\left[T: H^{\prime}\right]_{r} \cdot\left[H^{\prime}: B\right]_{r}=[T: B]_{r}$ by Prop. 2 (c). Since $A / B^{\prime}$ is $h-q$-Galois, by the same reason, we have $\left[V_{A}\left(B^{\prime}\right): V_{A}(T)\right]=$ $\left[V_{A}^{2}(T): V_{A}^{2}\left(B^{\prime}\right)\right]$ and $\left[T: B^{\prime}\right]_{l}=\left[T: B^{\prime}\right]_{r}$. Combining those above with the fact that $A$ is $B^{\prime} \cdot V^{\prime}-A$-irreducible (Th. 1 ), it follows at once $\left[B^{\prime}: B\right]_{r}$ $=\left[B^{\prime}: B\right]_{l} \geq\left[V: V_{A}\left(B^{\prime}\right)\right]=\left[V_{A}^{2}\left(B^{\prime}\right): H\right]$ by Prop. 1 (b). Now, we shall prove $\left[B^{\prime}: H \cap B^{\prime}\right]=\left[V_{A}^{2}\left(B^{\prime}\right): H\right]$. If $H^{*}=\left(H \cap B^{\prime}\right)[\Delta]$ and $B^{*}=H^{*}\left[B^{\prime}\right]$ then $B^{*}$ is regular as an intermediate ring of $V_{A}^{2}\left(B^{\prime}\right) / B^{\prime}$ (Th. 4 and Prop. 2 (a)). Hence, Cor. 6 yields $\left[B^{*}: H \cap B^{*}\right]=\left[V_{A}^{2}\left(B^{*}\right): H\right]=\left[V_{A}^{2}\left(B^{\prime}\right): H\right]$. Recalling here that $\mathscr{S}=\mathbb{S}\left(V_{A}^{2}\left(B^{\prime}\right) / B^{\prime}\right)=\mathbb{S}\left(H\left[B^{\prime}\right] / B^{\prime}\right) \approx \mathbb{S}\left(H / H \cap B^{\prime}\right)$ by contraction (Th. 5), Prop. 2 (c) yields $\left[B^{*}: B^{\prime}\right]=\#\left(\mathfrak{S} \mid B^{*}\right)=\#\left(\mathfrak{g} \mid H^{*}\right)=\#(\mathfrak{I} \mid H$ $\left.\cap B^{*}\right)=\left[H \cap B^{*}: H \cap B^{\prime}\right]$, whence it follows $\left[B^{\prime}: H \cap B^{\prime}\right]=\left[B^{*}: H \cap B^{*}\right]$. We have proved therefore $\left[B^{\prime}: H \cap B^{\prime}\right]=\left[V_{A}^{2}\left(B^{\prime}\right): H\right]$.

Lemma 7. Let $A$ be $h-q$-Galois and left locally finite over $B$. If $A^{\prime}$ is an $f$ regular intermediate ring of $A / B$ then $A / A^{\prime}$ is left locally finite and $\left[A^{\prime}: H \cap A^{\prime}\right]_{l}$ $=\left[V: V_{A}\left(A^{\prime}\right)\right]$.

Proof. Let $N$ be an arbitrary $\$(H / B)$-invariant shade of $\Delta$. Then, by Th. 5 and Prop. $2(\mathrm{~b})$, we have $\left[N\left[A^{\prime}\right]: A^{\prime}\right]=\left[N: N \cap A^{\prime}\right]<\infty$ and $H \cap N\left[A^{\prime}\right]$ $=H \cap\left(N\left[H \cap A^{\prime}\right]\right)\left[A^{\prime}\right]=N\left[H \cap A^{\prime}\right]$. Since $H \cap A^{\prime}$ is also a regular intermediate ring of $A / B$ (Prop. 2 (a)), we obtain $\left[H \cap N\left[A^{\prime}\right]: H \cap A^{\prime}\right]=\left[N\left[H \cap A^{\prime}\right]: H\right.$ $\left.\cap A^{\prime}\right]=\left[N: N \cap A^{\prime}\right]=\left[N\left[A^{\prime}\right]: A^{\prime}\right]<\infty$ again by Th. 5 and Prop. 2 (b). We choose here a simple intermediate ring $B^{\prime}$ of $A^{\prime} / B$ with $\left[B^{\prime}: B\right]<\infty$ and $V_{A}\left(B^{\prime}\right)=V_{A}\left(A^{\prime}\right)$, and set $B^{*}=N\left[B^{\prime}\right]$. Then, $B^{*}$ is a regular subring of $A$ with $\left[B^{*}: B\right]<\infty$ as an intermediate ring of $V_{A}^{2}\left(B^{\prime}\right) / B^{\prime}$ (Th. 4 and Prop. 2). Recalling that $H\left[B^{*}\right]=V_{A}^{2}\left(B^{*}\right) \supseteq N\left[A^{\prime}\right] \supseteq B^{*} \supseteq 4$, Cor. 6 and Th. 8 imply $\left[N\left[A^{\prime}\right]: H \cap N\left[A^{\prime}\right]\right]_{l}=\left[B^{*}: H \cap B^{*}\right]=\left[V: V_{A}\left(B^{*}\right)\right]=\left[V: V_{A}\left(B^{\prime}\right)\right]<\infty$. Combining this with $\left[H \cap N\left[A^{\prime}\right]: H \cap A^{\prime}\right]=\left[N\left[A^{\prime}\right]: A^{\prime}\right]<\infty$, it follows at once $\left[A^{\prime}: H \cap A^{\prime}\right]_{l}=\left[N\left[A^{\prime}\right]: H \cap N\left[A^{\prime}\right]\right]_{l}=\left[V: V_{A}\left(B^{\prime}\right)\right]=\left[V: V_{A}\left(A^{\prime}\right)\right]$, which is the latter assertion. Next, we shall prove the first half. Here, without loss of generality, we may assume that $A^{\prime} \subseteq H$. For an arbitrary finite subset $F$ of $A$, we set $B_{1}=B[E, \Delta, F]$. Then, $\left[A^{\prime}\left[H \cap B_{1}\right]: A^{\prime}\right]<\infty$ by Prop. 2 and 
$\left[A^{\prime}\left[B_{1}\right]: A^{\prime}\left[H \cap B_{1}\right]\right]_{l}=\left[B_{1}: H \cap B_{1}\right]_{l} \leq\left[B_{1}: B\right]<\infty$ by Cor. 6. It follows therefore $\left[A^{\prime}[F]: A^{\prime}\right]_{l} \leq\left[A^{\prime}\left[B_{1}\right]: A^{\prime}\left[H \cap B_{1}\right]\right] \cdot\left[A^{\prime}\left[H \cap B_{1}\right]: A^{\prime}\right]<\infty$.

The next theorem contains evidently [6, Ths. 2 and 4$]$.

Theorem 9. Let $A$ be h-q-Galois and left locally finite over $B$. If $A^{\prime}$ is an f-regular intermediate ring of $A / B$ then $A$ is $h$-q-Galois, right $h$-q-Galois and locally finite over $A^{\prime}$ and $\left[A^{\prime}: H \cap A^{\prime}\right]=\left[V: V_{A}\left(A^{\prime}\right)\right]=\left[V_{A}^{2}\left(A^{\prime}\right): H\right]$.

Proof. To prove the first assertion, we may restrict our attention to the case that $A^{\prime} \subseteq H$. If $A^{\prime \prime}$ is a regular intermediate ring of $A / A^{\prime}$ with $\left[A^{\prime \prime}: A^{\prime}\right]_{l}$ $<\infty$ then, to be easily verified, $A^{\prime \prime}$ is $f$-regular. Since $A_{0}=A^{\prime \prime}[E, \Delta]$ is left finite over $A^{\prime}$ (Lemma 7), $\left(\$\left(A^{\prime \prime}, A / A^{\prime}\right) A_{r}=\left(\&\left(A_{0}, A / A^{\prime}\right) \mid A^{\prime \prime}\right) A_{r}\right.$ (Th. 6). And so, we see that it suffices to prove that $\operatorname{Hom}_{A l^{\prime}}\left(A^{\prime \prime}, A\right)=\mathbb{G}\left(A^{\prime \prime}, A / A^{\prime}\right) A_{r}$ for each intermediate ring $A^{\prime \prime}$ of $A / A^{\prime}[E, \Delta]$ with $\left[A^{\prime \prime}: A^{\prime}\right]_{l}<\infty$. By Th. 4 and Prop. 2 (a), $H^{\prime \prime}=A^{\prime \prime} \cap H$ is a simple subring of $H$. As $\mathbb{B}(H / B) \mid H^{\prime \prime}=\mathbb{B}(H$, $A / B)\left|H^{\prime \prime}=\mathbb{B}\left(V_{A}^{2}\left(A^{\prime \prime}\right), A / B\right)\right| H^{\prime \prime}$ (Ths. 4 and 6), it follows $\mathbb{B}\left(H / A^{\prime}\right) \mid H^{\prime \prime}=$ $\$\left(V_{A}^{2}\left(A^{\prime \prime}\right), A / A^{\prime}\right) \mid H^{\prime \prime}$ (Prop. $2(\mathrm{~b})$ ). Recalling that $\$\left(H / A^{\prime}\right) H_{r}$ is dense in $\operatorname{Hom}_{A l^{\prime}}(H, H)$ (Prop. 2) and that $\left[H^{\prime \prime}: A^{\prime}\right]<\infty$ (Prop. 2 (c) or Th. 8), we have then $\operatorname{Hom}_{A l^{\prime}}\left(H^{\prime \prime}, H\right)=\left(\mathbb{S}\left(V_{A}^{2}\left(A^{\prime \prime}\right), A / A^{\prime}\right) \mid H^{\prime \prime}\right) H_{r}=\oplus_{1}^{s}\left(\sigma_{i} \mid H^{\prime \prime}\right) H_{r}$ with some $\sigma_{i} \in \mathbb{S}\left(V_{A}^{2}\left(A^{\prime \prime}\right), A / A^{\prime}\right)$ (Lemma 2). Since $\sigma_{i}\left|H^{\prime \prime} \neq \sigma_{j}\right| H^{\prime \prime}(i \neq j)$, irreducible $\left(\sigma_{i} \mid A^{\prime \prime}\right) A_{r}$ is not $A_{r}^{\prime \prime}-A_{r}$-isomorphic to $\left(\sigma_{j} \mid A^{\prime \prime}\right) A_{r}$ (Lemma 2), which implies $\sum_{1}^{s}\left(\sigma_{i} \widetilde{V} \mid A^{\prime \prime}\right) A_{r}=\oplus_{1}^{s}\left(\sigma_{i} \widetilde{V} \mid A^{\prime \prime}\right) A_{r} . \quad$ By [4, Lemma 1.5] and Th. 8, there holds $\left[\left(\tilde{V} \mid A^{\prime \prime}\right) A_{r}: A_{r}\right]_{r}=\left[V: V_{A}\left(A^{\prime \prime}\right)\right]=\left[V_{A}^{2}\left(A^{\prime \prime}\right): H\right]$. On the other hand, the same reason together with Ths. 4 and 6 implies $\infty>\left[\left(\sigma_{i} \tilde{V} \mid A^{\prime \prime}\right) A_{r}: A_{r}\right]_{r}=$ $\left[\left(\tilde{V} \mid A^{\prime \prime} \sigma_{i}\right) A_{r}: A_{r}\right]_{r}=\left[V: V_{A}\left(A^{\prime \prime} \sigma_{i}\right)\right]=\left[V_{A}^{2}\left(A^{\prime \prime} \sigma_{i}\right): H\right]=\left[\left(H\left[A^{\prime \prime}\right]\right)_{\sigma_{i}}: H \sigma_{i}\right]=$ $\left[V_{A}^{2}\left(A^{\prime \prime}\right): H\right]$. It follows therefore $\left[\left(\sigma_{i} \tilde{V} \mid A^{\prime \prime}\right) A_{r}: A_{r}\right]_{r}=\left[V: V_{A}\left(A^{\prime \prime}\right)\right]$, whence we obtain $\left[\sum_{1}^{s}\left(\sigma_{i} \tilde{V} \mid A^{\prime \prime}\right) A_{r}: A_{r}\right]_{r}=s \cdot\left[V: V_{A}\left(A^{\prime \prime}\right)\right]=\left[\operatorname{Hom}_{i^{\prime} l}\left(H^{\prime \prime}, H\right): H_{r}\right]_{r} \cdot$ $\left[V: V_{A}\left(A^{\prime \prime}\right)\right]=\left[H^{\prime \prime}: A^{\prime}\right] \cdot\left[A^{\prime \prime}: H^{\prime \prime}\right]_{l}=\left[A^{\prime \prime}: A^{\prime}\right]_{l}$ by Lemma 7 . We have proved therefore $\operatorname{Hom}_{A^{\prime} l}\left(A^{\prime \prime}, A\right)=\sum_{1}^{s}\left(\sigma_{i} \widetilde{V} \mid A^{\prime \prime}\right) A_{r}=\mathbb{B}\left(A^{\prime \prime}, A / A^{\prime}\right) A_{r}$ by $\left(2^{*}\right)$, and $A / A^{\prime}$ is locally finite by Lemma 7 and Th. 8. The final equalities are now direct consequences of Lemma 7 and Th. 8 , for $A^{\prime} \cap H$ is $f$-regular. In particular, noting that $\left[A^{\prime}: H \cap A^{\prime}\right]=\left[V: V_{A}\left(A^{\prime}\right)\right]$, we can repeat a symmetric argument to see that $A / A^{\prime}$ is right $h-q$-Galois.

COROLlary 7. The following conditions are equivalent to each other:

(Q) $A / B$ is $h$-q-Galois and left locally finite. 
$\left(\mathrm{Q}^{\prime}\right) A / B$ is right $h-q-$ Galois and right locally finite.

Combining Th. 9 with Th. 7, we readily obtain the following:

Corollary 8. Let $A$ be $h-q$-Galois and left locally finite over $B$ and $[A$ : $H]_{l} \leq\left\{\oint_{0}\right.$. If $A^{\prime}$ is an f-regular intermediate ring of $A / B$ then $A / A^{\prime}$ is $h$-Galois and locally finite.

Now, we shall add to Prop. 4 other equivalent conditions to complete [2, Th. 1].

Proposition 7. Let $B$ be a regular subring of $A . \quad A / B$ is $h$-Galois and left locally finite over $B$ if and only if any of the following conditions is satisfied:

(D) $A$ is Galois and left locally finite over $B, H$ is simple, and $\left[V_{A}^{2}\left(B^{\prime}\right): H\right]_{l}$ $=\left[V: V_{A}\left(B^{\prime}\right)\right]_{r}$ for every regular intermediate ring $B^{\prime}$ of $A / B$ with $\left[B^{\prime}: B\right]_{l}$ $<\infty$.

$\left(\mathrm{D}^{\prime}\right) A$ is Galois and right locally finite over $B, H$ is simple, and $\left[V_{A}^{2}\left(B^{\prime}\right)\right.$ : $H]_{r}=\left[V: V_{A}\left(B^{\prime}\right)\right]_{l}$ for every regular intermediate ring $B^{\prime}$ of $A / B$ with $\left[B^{\prime}: B\right]_{r}$ $<\infty$.

(E) $A$ is left locally finite over $B$ and Galois over every regular subring left finite over $B, H$ is simple, and $\left[A^{\prime}: H\right]_{l}=\left[V: V_{A}\left(A^{\prime}\right)\right]_{r}$ for every regular intermediate ring $A^{\prime}$ of $A / H$ with $\left[A^{\prime}: H\right]_{l}<\infty$.

( $\left.\mathrm{E}^{\prime}\right) A$ is right locally finite over $B$ and Galois over every regular subring right finite over $B, H$ is simple, and $\left[A^{\prime}: H\right]_{r}=\left[V: V_{A}\left(A^{\prime}\right)\right]_{l}$ for every regular intermediate ring $A^{\prime}$ of $A / H$ with $\left[A^{\prime}: H\right]_{r}<\infty$.

Proof. Since $(\mathrm{A}) \Longrightarrow(\mathrm{D})$ and (E) is evident by Cor. 1 and Th. 9, it is left to prove the converse. Now, let $T$ be an arbitrary intermediate ring of $A / B$ $[E, \Delta]$ with $[T: B]_{l}<\infty$, and set $T^{\prime}=J(\&(T), A)$ and $H^{\prime}=H \cap T^{\prime}$. Then, $\left[H^{\prime}: B\right]<\infty$ by Prop. 3 (b). Noting that $A$ is $H^{\prime}[T]-A$-irreducible, Prop. 1 (b) yields $\infty>\left[H^{\prime}[T]: H^{\prime}\right]_{l} \geq\left[V_{A}\left(H^{\prime}\right): V_{A}\left(H^{\prime}[T]\right)\right]_{r}=\left[V: V_{A}\left(T^{\prime}\right)\right]_{r}$, whence it follows $\left[T^{\prime}: H^{\prime}\right]_{l} \geq\left[V: V_{A}\left(T^{\prime}\right)\right]_{r}$. In case (D), Lemma 3 yields then $\left[T^{\prime}\right.$ : $\left.H^{\prime}\right]_{l}=\left[H \cdot T^{\prime}: H\right]_{l} \leq\left[V_{A}^{2}\left(T^{\prime}\right): H\right\rceil_{l}=\left[V_{A}^{2}(T): H\right]_{l}=\left[V: V_{A}(T)\right]_{r}=[V:$ $\left.V_{A}\left(T^{\prime}\right)\right]_{r}$. Hence, we have $\left[T^{\prime}: H^{\prime}\right]_{l}=\left[V: V_{A}\left(T^{\prime}\right)\right]_{r}<\infty$, so that it follows $\operatorname{Hom}_{B_{l}}\left(T^{\prime}, A\right)=\left(\$ \mid T^{\prime}\right) A_{r}$ by Prop. 3 (a), which proves (D) $\Rightarrow(\mathrm{A})$. Now, we shall prove $(\mathrm{E}) \Longrightarrow(\mathrm{A})$. If $N$ is an arbitrary $(\dot{B}(H / B)$-invariant shade of $H^{\prime}$, then $\mathbb{B}(T) \mid N[T]$ and $\mathscr{B}(T) \mid N$ are (outer) Galois groups of $N[T] / T$ and $N / H^{\prime}$, respectively. There holds then $\left[N: H^{\prime}\right]=\#(\$(T) \mid N)=\#(\mathbb{B}(T) \mid N[T])$ 
$=[N[T]: T]$ (Prop. $2(\mathrm{c})$ ), and so Lemma 3 yields $\left[N \cdot T: H^{\prime}\right]_{l}=[N \cdot T: N]_{l}$ $\cdot\left[N: H^{\prime}\right]=\left[T: H^{\prime}\right]_{l} \cdot[N[T]: T]=\left[N[T]: H^{\prime}\right]_{l}$, whence we obtain $N \cdot T=$ $N[T]$. We readily see then $H \cdot T$ is a regular intermediate ring of $A / H$ with $[H \cdot T: H]_{l}=\left[T: H^{\prime}\right]_{l}<\infty$. It follows therefore $\left[T: H^{\prime}\right]_{l}=[H \cdot T: H]_{l}=[V:$ $\left.V_{A}(T)\right]_{r}$, and we have $\operatorname{Hom}_{B_{l}}(T, A)=(\$ \mid T) A_{r}$ again by Prop. 3 (a).

We shall present here a notably short proof to $[4, \text { Lemma } 2.2]^{5}$.

Proposition 8. If $A$ is Galois and left locally finite over $B$ and $[V: C]<\infty$, then $A / B$ is (3-locally Galois.

Proof. By the validity of Prop. 6, it suffices to prove that $A / B$ is locally Galois. To be easily seen, ( $H$ is simple and $\left[V_{A}^{2}\left(B^{\prime}\right): H\right]_{l}=\left[V: V_{A}\left(B^{\prime}\right)\right]_{r}$ for each regular intermediate ring $B^{\prime}$ of $A / B$ with $\left[B^{\prime}: B\right]_{l}<\infty . A / B$ is therefore $h$-Galois by Prop. 7. We set here $V=\sum U g_{p q}$, where $I^{\prime}=\left\{g_{p q}\right.$ 's $\}$ is a system of matrix units and $U=V_{V}\left(I^{\prime}\right)$ a division ring. Now, let $B^{\prime}$ be an arbitrary intermediate ring of $A / B[E, \Gamma]$ with $\left[B^{\prime}: B\right]_{l}<\infty$. Since $J\left(\& \mid B^{\prime}, B^{\prime}\right)$ $=B$, there exists a finite subset $\mathfrak{F}$ of $\mathbb{B}$ with $J\left(\mathfrak{F} \mid B^{\prime}, B^{\prime}\right)=B$. If $N$ is an arbitrary $\mathbb{S}(H / B)$-invariant shade of $B^{\prime}\left[\bigcup_{\sigma \in \mathfrak{F}} B^{\prime} \sigma\right] \cap H$ then $B^{\prime}\left[\cup B^{\prime} \sigma\right]$ is contained in the simple ring $M=N\left[B^{\prime}\right]$ (Th. 5 and Prop. $2(\mathrm{~b})$ ). And so, $\mathscr{g}=\mathscr{S}\left(B^{\prime}\right)[\mathfrak{F}]$ induces an automorphism group of $M$. Since $J(\mathfrak{I} \mid M, M)=B$ and $V_{M}(B)$ is evidently simple, $M / B$ is Galois, which implies that $A / B$ is locally Galois.

We shall conclude this section with the following theorem, whose first assertion is [4, Lemma 4.2].

TheOREM 10. (a) If $A / B$ is locally Galois then $H$ is simple and for each finite subset $F$ of $A$ there exists a simple intermediate ring $A^{\prime}$ of $A / H[F]$ such that $\left[A^{\prime}: H\right]_{i}<\infty$ and $A^{\prime} / B$ is Galois, and conversely provided $A / B$ is left locally finite.

(b) If $A / B$ is locally Galois then so is $A / A^{\prime}$ for every f-regular intermediate ring $A^{\prime}$ of $A / B$.

5) The proof of Prop. 8 given in [4] enabled us moreover to see that there exists a Galois group $\mathfrak{H}$ of $A / B$ with the property that ( $\mathfrak{S}[\mathfrak{F}], A / B$ ) is l.f.d. for each finite subset $\mathfrak{F}$ of $\mathbb{S}$, which was needed only to prove the following: If $A$ is Galois and left locally finite over $B$ and $[V: C]<\infty$, then every $(*)$-regular subgroup of $B$ is regular. However, in [2] and [10], we have proved directly an extension of the last proposition (cf. also Th. 11 (a)), 
Proof. (a) Let $V=\sum U g_{p q}$, where $\Gamma=\left\{g_{p q}\right.$ 's $\}$ is a system of matrix units and $U=V_{V}(\Gamma)$ a division ring. If $B^{\prime}$ is an arbitrary shade of $B[E, \Gamma]$, then $A^{\prime}=V_{A}^{2}\left(B^{\prime}\right)=H\left[B^{\prime}\right]=\cup N_{\alpha}\left[B^{\prime}\right]$, where $N_{\alpha}$ ranges over all the $\mathbb{B}(H / B)$-invariant shades. Now, let $B^{\prime \prime}$ be a shade of $N_{\alpha}\left[B^{\prime}\right]$, and $\mathbb{S}^{\prime}=\left\{\sigma \in \mathbb{B}\left(B^{\prime \prime} / B\right) ; B^{\prime} \sigma=B^{\prime}\right\}$. Then, noting that $\left(B\left(B^{\prime} / B\right) \subseteq \mathbb{S}^{\prime} \mid B^{\prime}\right.$, Th. 5 together with Lemma 6 and Prop. 2 proves that $N_{\alpha}\left[B^{\prime}\right] / B$ is Galois. Hence, $A^{\prime} / B$ is locally Galois, and so it is Galois by Th. 7, for $\left[V_{A^{\prime}}(B): V_{A^{\prime}}\left(A^{\prime}\right)\right]=\left[V_{A^{\prime}}(H): V_{A^{\prime}}\left(A^{\prime}\right)\right] \leq\left[A^{\prime}: H\right]_{l}<\infty$ (Prop. 1). And, by the fact used just above, the converse part will be an easy consequence of Prop. 8.

(b) If $B^{\prime}$ is an intermediate simple ring of $A^{\prime} / B$ with $\left[B^{\prime}: B\right]_{l}<\infty$ and $V_{A}\left(B^{\prime}\right)=V_{A}\left(A^{\prime}\right)$, then $A / B^{\prime}$ is locally Galois. And so, by (a), for each finite subset $F$ of $A$ there exists a simple intermediate ring $A^{\prime \prime}$ of $A / V_{A}^{2}\left(B^{\prime}\right)[F]$ such that $A^{\prime \prime} / B^{\prime}$ is Galois and $\left[V_{A^{\prime \prime}}\left(B^{\prime}\right): V_{A^{\prime \prime}}\left(A^{\prime \prime}\right)\right] \leq\left[A^{\prime \prime}: V_{A}^{2}\left(B^{\prime}\right)\right]_{l}<\infty$. Prop. 8 implies then that $A^{\prime \prime} / B^{\prime}$ is $\left(A^{\prime \prime}\left(A^{\prime \prime} / B^{\prime}\right)\right.$-locally Galois. Since $A^{\prime \prime} / A^{\prime}$ is $h$-Galois and locally finite by Cor. $8, A^{\prime \prime} / A^{\prime}$ is locally Galois again by Prop. 8. We have proved therefore $A / A^{\prime}$ is locally Galois.

4. $\left(*_{f}\right)$-Regular Subgroups. By the validity of Ths. 4, 9 and Cor. 2 (and Lemma 3 if necessary), the proofs of Lemmas 2, 3 of [10] are applicable without any change to those of the following lemmas.

Lemma 8. Let $A$ be $h-q$-Galois and left locally finite over $B$, and $\mathscr{B}^{\prime} a\left(*_{f}\right)$ regular subgroup of $\$$. If $A^{\prime}=J\left(\$^{\prime}, A\right)$ then $\left[A^{\prime}: H \cap A^{\prime}\right]_{l}<\infty$.

Lemma 9. Let $A$ be $h-q$-Galois and left locally finite over $B$, and $V^{\prime}$ a simple subring of $V$ with $\left[V: V^{\prime}\right]_{r}<\infty$. If $V_{A}\left(V_{A}\left(V^{\prime}\right)[F]\right) \subseteq V^{\prime}$ for some finite subset $F$ of $A$ then $V_{A}\left(V^{\prime}\right)$ is a simple ring.

The first assertion of the following theorem contains [10, Th. 2].

Theorem 11. Let $A$ be $h$-q-Galois and left locally finite over $B$, and (B' a $\left(*_{f}\right)$-regular subgroup of $\$$ with $A^{\prime}=J\left(\mathbb{S}^{\prime}, A\right)$.

(a) $\mathbb{S}^{\prime}$ is f-regular (i.e. $A^{\prime}$ is simple) and dense in $\mathbb{B}\left(A^{\prime}\right)$.

(b) $\tilde{V} \cdot \mathrm{Cl}\left(\dot{S}^{\prime}{ }^{6)}=\mathbb{B}\left(H \cap A^{\prime}\right)\right.$.

(c) If $\mathfrak{S}$ is an open subgroup of $\mathbb{B}$ then $\left(\mathrm{Cl}^{\prime} \mathbb{S}^{\prime}:\left(\mathfrak{S} \cap \mathrm{Cl}^{\prime}\right) \widetilde{V}\left(\mathfrak{S}^{\prime}\right)<\infty\right.$.

6) $\mathrm{Cl}\left(\mathrm{S}^{\prime}\right.$ is the topological closure of $\mathbb{S}^{\prime}$ in (5). 
Proof. One may remark here that $H^{\prime}=H \cap A^{\prime}$ is $f$-regular (Th. 4 and Prop. 2). As $\left[V: V_{\mathbb{S}^{\prime}}\right]_{r}<\infty$ and $V_{\mathbb{S}^{\prime}}=V_{A}^{2}\left(V_{\mathbb{S}^{\prime}}\right), V_{A}^{2}\left(A^{\prime}\right)=V_{A}\left(V_{\mathbb{S}^{\prime}}\right)$ is simple by Lemma 9 . Further, by Lemma 8 , there holds $\left[A^{\prime}: H^{\prime}\right]_{l}<\infty$. Since $A / H^{\prime}$ is locally finite (Th. 9), $V_{\mathrm{r}^{\prime}}^{2}\left(A^{\prime}\right)\left(A^{\prime}\right)$ coincides with the center of $V_{A}^{2}\left(A^{\prime}\right)$ and $J\left(\$^{\prime} \mid V_{A}^{2}\left(A^{\prime}\right), V_{A}^{2}\left(A^{\prime}\right)\right)=A^{\prime}$, [10, Lemma 1] proves that $A^{\prime}$ is simple. And so, $A / A^{\prime}$ is $h-q$-Galois and locally finite (Th. 9 ). If $T$ is an arbitrary intermediate ring of $A / A^{\prime}[E]$ with $\left[T: A^{\prime}\right]<\infty$, then $A$ is $T$-A-irreducible and $\left[T: V_{A}^{2}\left(A^{\prime}\right) \cap T\right]=\left[V_{A}\left(A^{\prime}\right): V_{A}(T)\right](T h .8)$. Hence, $A / A^{\prime}$ is $h$-Galois and $\left(B^{\prime}\right.$ is dense in $\mathbb{B}\left(A^{\prime}\right)$ by Prop. 3 (a), which completes the proof of (a). Recalling here that $\left[T: H^{\prime}\right]_{l}=\left[T: A^{\prime}\right]_{l} \cdot\left[A^{\prime}: H^{\prime}\right]_{l}<\infty$ (Lemma 8), for each $\sigma \in \operatorname{Cl}(\bar{V} \cdot$ $\left.\mathrm{Cl}^{\prime}\right)$ we can find such an element $\tau \in \hat{V} \cdot \mathrm{Cl}\left(\mathcal{S}^{\prime}\right.$ that $\tau|T=\rho| T$. And then $\sigma \tau^{-1}$ is contained in $\mathbb{B}(T) \subseteq\left(B\left(A^{\prime}\right)=\mathrm{Cl} \mathbb{S}^{\prime}\right.$ by (a). Hence, $\sigma$ is contained in $\tilde{V} \cdot \mathrm{Cl} \mathbb{B}^{\prime}$, which means that $\tilde{V} \cdot \mathrm{Cl} \mathbb{B}^{\prime}$ is a closed $\left(*_{f}\right)$-regular subgroup of $\mathbb{B}$ with $J(\tilde{V} \cdot$ $\left.\mathrm{C} 1 \mathbb{S}^{\prime}, A\right)=H^{\prime}$. Accordingly, ( $\left.\mathrm{b}\right)$ is a consequence of $(\mathrm{a})$. Finally, we shall prove $(\mathrm{c})$. Since $J\left(\mathrm{Cl}_{\mathbb{S}^{\prime}}, A\right)=A^{\prime}$ and $V_{\mathrm{CI}_{(} \mathfrak{S}^{\prime}}=V_{\mathbb{S}^{\prime}}$, it suffices to prove our assertion for closed $\mathbb{B}^{\prime}=\mathbb{S}\left(A^{\prime}\right)$. Moreover, without loss of generality, we may assume that $\mathscr{H}=\mathscr{S}\left(B^{\prime}\right)$ for some intermediate ring $B^{\prime}$ of $A / B[E]$ with $\left[B^{\prime}\right.$ : $B]_{l}<\infty$. If $T=A^{\prime}\left[B^{\prime}\right]$ (finite over $A^{\prime}$ ) then $\mathbb{S}^{\prime}(T)$ is a closed $\left(*_{f}\right)$-regular subgroup of $\mathcal{S}^{\prime}$ with $J\left(\mathbb{S}^{\prime}(T), A\right)=T$ by Cor. 1 or [5, Theorem 1]. And so, by (b), it follows $\left(\mathfrak{g} \cap \mathbb{S}^{\prime}\right) \widetilde{V}_{\mathfrak{B}^{\prime}}=\mathbb{S}^{\prime}(T) \widehat{V_{A}\left(A^{\prime}\right)}=\mathbb{B}^{\prime}\left(V_{A}^{2}\left(A^{\prime}\right) \cap T\right)$. Hence, by Th. 4 and Prop. 2 (c), we obtain $\left(\mathbb{S}^{\prime}:\left(\mathfrak{S} \cap \mathbb{S}^{\prime}\right) \widetilde{V}_{\mathbb{S}^{\prime}}\right)=\left(\mathbb{S}^{\prime}: \mathbb{S}^{\prime}\left(V_{A}^{2}\left(A^{\prime}\right) \cap T\right)\right)=$ $\#\left(\$^{\prime} \mid V_{A}^{2}\left(A^{\prime}\right) \cap T\right)=\left[V_{A}^{2}\left(A^{\prime}\right) \cap T: A^{\prime}\right]<\infty$.

As a direct consequence of Th. 11 (a) and Cors. 1, 8, we readily obtain the following theorem.

Theorem 12. If $A$ is $h-q$-Galois and left locally finite over $B$ and $[A: H]_{l}$ $\leq \$ \aleph_{0}$ then there exists a 1-1 dual correspondence between closed $\left(*_{f}\right)$-regular subgroups and f-regular intermediate rings of $A / B$, in the usual sense of Galois theory.

Remark 3. Evidently, Th. 12 is nothing but [2, Th. 5], and the assumption cited in Th. 12 is the best one obtained by now to allow the existence of Galois correspondence.

Let $A / B$ be $h-q$-Galois and left locally finite. If $T$ is an intermediate ring of $A / B$ left finite over $B$ such that $A$ is $T$-A-irreducible and $J(B(T), A)=T$, then $T$ is a simple ring by Th. 11 (a). In particular, if $A / B$ is $h$-Galois then 
the assumption $J(\mathbb{S}(T), A)=T$ is automatically enjoyed by [5, Th. 1] (cf. $[2$, Cor. 6$])$. The next will be an easy consequence of the above remark, $\mathrm{Th}$. 1 and [4, Lemma 1.1].

Proposition 9. Let $A / B$ be locally $h$-Galois and left locally finite. If $V$ is a division ring then every intermediate ring of $A / B$ is simple.

Remark 4. Let $A$ be left algebraic over $B$ (that is, $[B[a]: B]_{l}<\infty$ for every $a \in A$ ). If every intermediate ring of $A / B$ left finite over $B$ is a simple ring then $V$ is a division ring. In fact, for an arbitrary non-zero element $v \in V, B[v]$ is a simple ring, and so the center of $B[v]$ is a field. Hence, $v$ belonging to the center of $B[v]$ is regular and $v^{-1}$ is contained in $V$.

We shall conclude our study with the following (cf. [2, Th. 2]).

Theorem 13. Let $A$ be $h-q-G a l o i s$ and left locally finite over $B$, and $\mathbb{S}^{\prime}$ an $N$-regular subgroup of $\mathbb{B}$. Then, $\mathbb{S}^{\prime}$ is $\left(*_{f}\right)$-regular if and only if $\left[V: I\left(\mathbb{S}^{\prime}\right)\right]_{r}$ $<\infty, V_{A}^{2}\left(I\left(\mathbb{S}^{\prime}\right)\right)=I\left(\mathbb{S}^{\prime}\right)=I\left(\mathrm{Cl} \mathbb{S}^{\prime}\right)$ and $\left(\mathrm{Cl}^{\mathbb{S}^{\prime}}:\left(\mathscr{S} \cap \mathrm{Cl}\left(\mathbb{S}^{\prime}\right) I\left(\mathbb{S}^{\prime}\right)\right)<\infty\right.$ for every open subgroup 5 of $(\mathbb{S}$.

Proof. If $\mathbb{B}^{\prime}$ is $\left(*_{f}\right)$-regular then $I\left(\mathbb{S}^{\prime}\right)$ coincides with $V_{\mathbb{S}}$, so that the only if part is obvious by Th. 11. To prove the if part, we may restrict our proof to the case that $\mathbb{S}^{\prime}$ is closed. By Th. $11(\mathrm{a}), V_{A}\left(I\left(\mathbb{S}^{\prime}\right)\right)$ is simple and there exists a finite subset $F$ of $V_{A}\left(I\left(\mathbb{B}^{\prime}\right)\right)$ with $V_{A}(B[F])=I\left(\mathbb{B}^{\prime}\right)$. If we set $\mathscr{S}=$

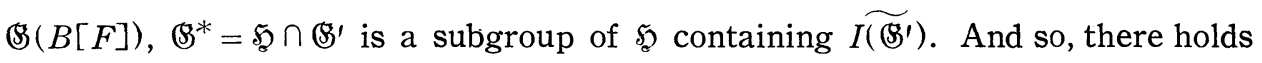
$B[F] \subseteq J\left(\mathbb{S}^{*}, A\right) \subseteq V_{A}\left(I\left(\mathbb{S}^{\prime}\right)\right)$, which implies $I\left(\mathbb{S}^{\prime}\right)=V_{A}(B[F]) \supseteq V_{\mathscr{S}} \supseteq V_{A}^{2}\left(I\left(\mathbb{S}^{\prime}\right)\right)$ $=I\left(\mathbb{S}^{\prime}\right)$. We see therefore $\mathbb{B}^{*}$ is a closed $\left(*_{f}\right)$-regular subgroup of $\mathbb{B}$ with $V_{\mathscr{S}}=I\left(\mathbb{S}^{\prime}\right)$. By assumption, $\left(\mathbb{B}^{\prime}: \mathbb{B}^{*}\right)<\infty: \mathbb{S}^{\prime}=\cup_{1}^{m} \mathbb{S}^{*} \sigma_{i}$. Now, we set $A^{*}$ $=J\left(\mathbb{S}^{*}, A\right)$ and $A^{\prime}=J\left(\mathbb{S}^{\prime}, A\right)$. Then $\mathbb{S}^{*}=\mathbb{B}\left(A^{*}\right)$ and $A$ is $h$-Galois and locally finite over $A^{*}$ (Th. 11 (a) and its proof). And hence, by Th. 4 and Prop. 2, $A^{* *}=A^{*}\left[\cup_{1}^{m} A^{*} \sigma_{i}\right]$ is a $B^{\prime}$-invariant simple ring as an intermediate ring between $V_{A}^{2}\left(A^{*}\right)=V_{A}\left(V_{\mathscr{S}^{*}}\right)=V_{A}\left(I\left(\mathbb{S}^{\prime}\right)\right)$ and $A^{*}$. If an element $\sigma \in \mathbb{S}^{\prime}$ induces an inner automorphism in $A^{* *}: \sigma\left|A^{* *}=\widetilde{v}\right| A^{* *}\left(v \in V_{A^{* *}}\left(A^{\prime}\right)\right)$ then $\sigma \mid \mathfrak{g} \cap A^{*}=1$, and so $\sigma$ is contained in $\mathbb{B}\left(H \cap A^{*}\right)=\mathbb{B}^{*} \widetilde{V}$ ( Th. $\left.11(\mathrm{~b})\right): \sigma=\tau \widetilde{u}\left(\tau \in \mathbb{S}^{*}, \tilde{u} \in \tilde{V}\right)$. But then, $\tau^{-1} \sigma=\tilde{u} \in \mathbb{B}^{\prime} \cap \tilde{V}=\widetilde{I\left(\mathbb{S}^{\prime}\right)}$ implies $\sigma \in \tau I\left(\mathbb{B}^{\prime}\right) \subseteq \mathbb{B}^{*}$, so that $v$ is contained in $V_{A^{* *}}\left(A^{*}\right)=V_{A^{* *}}\left(A^{* *}\right)$. Hence, $\sigma\left|A^{* *}=\widetilde{v}\right| A^{* *}=1$, which means $\mathcal{B}^{\prime} \mid A^{* *}$ is an outer group of finite order. Accordingly, as is well-known, $A^{* *}$ is outer Galois and finite over the simple ring $A^{\prime}$. Moreover, noting that $\mathbb{S}^{*}=\mathbb{S}^{*}(\widetilde{V}$ 
$\left.\cap \mathbb{S}^{\prime}\right)=\mathbb{S}^{*} \widetilde{V} \cap \mathbb{B}^{\prime}=\mathbb{S}^{\prime}\left(H \cap A^{*}\right) \cap \mathbb{S}^{\prime}=\mathbb{S}^{\prime}\left(H \cap A^{*}\right)$, we obtain $\left[A^{*}: A^{\prime}\right]=\#\left(\mathbb{S}^{\prime} \mid A^{*}\right)$ $=\left(\mathbb{B}^{\prime}: \mathbb{B}^{*}\right)=\#\left(\dot{B}^{\prime} \mid A^{\prime}\left[H \cap A^{*}\right]\right)=\left[A^{\prime}\left[H \cap A^{*}\right]: A^{\prime}\right]$ by Prop. 2 (c), whence there holds $A^{*}=A^{\prime}\left[H \cap A^{*}\right]$. We see therefore our assertion $I\left(\mathbb{S}^{\prime}\right)=V_{\mathfrak{G}^{*}}=V_{\mathfrak{S}^{\prime}}$.

\section{References}

[1] S. Eilenberg and N. Steenrod, Foundations of algebraic topology, Princeton (1952).

[2] T. Nagahara, On Galois conditions and Galois groups of simple rings, Trans. Amer. Math. Soc., 116 (1965), 417-434.

[ 3 ] T. Nagahara and H. Tominaga, On Galois and locally Galois extensions of simple rings, Math. J. Okayama Univ., 10 (1961), 143-166.

[4] T. Nagahara and H. Tominaga, On Galois theory of simple rings, Math. J. Okayama Univ., 11 (1963), 79-117.

[5] T. Nagahara and H. Tominaga, Some theorems on Galois theory of simple rings, J. Fac. Sci. Hokkaido Univ., Ser. I, 17 (1963), 1-13.

[6] T. Nagahara and H. Tominaga, On quasi-Galois extensions of division rings, J. Fac. Sci. Hokkaido Univ., Ser. I, 17 (1963), 73-78.

[ 7 ] T. Nakayama, Galois theory of simple rings, Trans. Amer. Math. Soc., 73 (1952), 276-292.

[8] N. Nobusawa and H. Tominaga, On Galois theory of division rings III, Math. J. Okayama Univ., 10 (1960), 67-73.

[9] H. Tominaga, Galois theory of simple rings II, Math. J. Okayama Univ., 6 (1957), 153-170.

[10] H. Tominaga, On Nagahara's theorem, J. Fac. Sci. Hokkaido Univ., Ser. I, 18 (1965), 153-157.

Department of Mathematics,

Hokkaido University 